\title{
Natural enemy enhancement and botanical insecticide source: a review of dual use companion plants
}

\author{
Blankson W. Amoabeng ${ }^{1,2} \cdot$ Anne C. Johnson ${ }^{1,3} \cdot$ Geoff M. Gurr $^{1,3,4}$
}

Received: 6 November 2018 / Accepted: 20 December 2018 / Published online: 18 January 2019

(c) The Author(s) 2019

\begin{abstract}
Intensive agriculture, which is associated with heavy inputs of synthetic insecticides, has serious ecological impacts, leading to loss of vital ecosystem services including insect-mediated pest suppression. In recent years, efforts have been made towards obtaining safer options to chemical insecticides for sustainable pest management. Habitat manipulation is a part of conservation biological control which aims at providing floral resources, alternative prey and shelter to predators and parasitoids to enhance and sustain natural pest suppression. The use of plant extracts as botanical insecticides is also an important provisioning ecosystem service. Selection of plant species for habitat manipulation has focused mainly on plants with suitable floral qualities to support natural enemies. To increase the benefits, habitat manipulation plants that can provide multiple ecosystem services in addition to floral resources would be an ideal. In this review, we focus on the potential of achieving the dual ecosystem services of bioinsecticidal source plants in addition to the provision of floral resources from selected plant species. Our literature search found 283 plants species from 44 plant families that have been involved in habitat manipulation studies. Fifteen of these plant families have species that have been exploited for their insecticidal properties. Three families, Apiaceae, Asteraceae and Lamiaceae, have the largest number of species that have been used for both habitat manipulation and botanical insecticides. Of the four most popular habitat manipulation plants, alyssum Lobularia maritime (L.) Desv. (Brassicaceae), buck wheat Fagopyrum esculentum Moench (Polygonaceae), coriander Coriandrum sativum L. (Apiaceae) and phacelia Phacelia tanacetifolia Benth. (Boraginaceae), buckwheat and coriander have been used for insecticidal purposes whilst no records exist of phacelia and alyssum as botanical insecticide species. There is great potential for identifying plant species that can support natural enemies as well as providing potent plant extracts as botanical insecticides by selecting species from the Apiaceae, Asteraceae and Lamiaceae families.
\end{abstract}

Keywords Habitat manipulation · Conservation biological control · Biopesticides · Dual ecosystem services

Geoff M. Gurr

ggurr@csu.edu.au

1 School of Agriculture and Wine Sciences, Charles Sturt University, Orange Campus, Orange, NSW, Australia

2 Council for Scientific and Industrial Research (CSIR), Crops Research Institute, Kumasi, Ghana

3 Graham Centre for Agricultural Innovation (New South Wales Department of Primary Industries and Charles Sturt University), Orange Campus, Orange, NSW, Australia

4 Institute of Applied Ecology, Fujian Agriculture and Forestry University, Fuzhou, Fujian, China

\section{Introduction}

Agriculture globally is facing many challenges including climate change, biodiversity loss and rising demands for food production (Deutsch et al. 2018; Rockstrom et al. 2009; Tilman et al. 2011). In response to these challenges, a growing volume of research is contributing towards a redesign of agricultural systems that provide nutritious food for all healthy and resilient ecosystems (Bommarco et al. 2013; Pretty et al. 2018; Struik and Kuyper 2017; Tilman et al. 2011). Evidence is growing that a sustainable intensification of agriculture can be achieved by combining scientific and farmer knowledge to develop ecologically and agronomically compatible practices (Pretty et al. 2018). Integrated pest management (IPM) is an example of redesigning intensive agricultural systems. Instead of 
relying principally on synthetic pesticides, IPM uses nonchemical or botanical insecticide measures to suppress pest population increase and a range of curative management tactics with synthetic pesticide use as last resort (Barzman et al. 2015). The declining availability of many pesticides due to resistance and deregistration, reflecting increasing awareness of their environmental and human health consequences, has driven changes towards ecologically based practices (Barzman et al. 2015; Borel 2017; Chagnon et al. 2015; Li et al. 2017; Sumon et al. 2018).

A central part of IPM is biological control in which natural enemies including parasitoids, predators and pathogens are introduced and/or promoted (Bale et al. 2008; Gurr et al. 2000a, 2018). Conservation biological control focuses on natural enemies already present in an agroecosystem and aims to maximize their impact on target pests by, for example, reducing the adverse effects of insecticide use (Begg et al. 2017; Ehler 1998). Habitat manipulation works in conjunction with conservation biological control and is used to provide conditions that promote natural enemies and suppress pest populations (Fiedler et al. 2008; Gurr et al. 2000b, 2017). This can include field level interventions such as establishing plants to provide floral resources, refuges and alternate hosts for natural enemies (Griffiths et al. 2008; Gurr et al. 2017). Plants that are selected for habitat manipulation have usually been studied for morphological and physiological floral characteristics that provide optimum benefits to natural enemies (Baggen et al. 1999; Balzan et al. 2014). Habitat manipulation tactics can extend beyond the field to include landscape features including riparian areas and treelines, although the effect of landscape features on crop pests is variable (Karp et al. 2018; Tscharntke et al. 2007).

Ecologically based pest management tactics such as conservation biological control have been shown to reduce the use of synthetic insecticides in a variety of cropping systems whilst maintaining or increasing crop yields and efforts are being made to up scale the practice globally (Pretty et al. 2018; Wyckhuys et al. 2013; Xu et al. 2017). Despite these advantages, however, uptake of conservation biological control on a wide scale is limited (Gurr et al. 2016). In cases where uptake has been strong, the vegetation used in habitat manipulation provides multiple ecosystem services rather than suppressing pests alone (Khan et al. 2006, 2012). To date, however, there is a major gap in knowledge about the possibility of habitat manipulation plants providing botanical insecticides. This is important because synthetic insecticides present significant risks to human health. Agricultural workers and consumers are at risk of being negatively affected by insecticide products, tank mixes, drift, residues and breakdown products, especially as a consequence of poor registration, storage and misuse (Eddleston et al. 2002). In agricultural areas where there are high illiteracy rates, and poor training and equipment, the impacts are especially high (Amoabeng et al. 2017; Williamson et al. 2008).

Many plants possess secondary metabolites such as alkaloids, phenols and terpenoids that can have insecticidal activity such as toxicity, repellency, feeding deterrence against insect pests (Koul 2004). Botanical insecticides, including extracts and essential oils of these plant species, have been used to protect crops against insect herbivory for many years (Belmain et al. 2012; Isman 2000, 2008). Synthetic insecticides often have lethal and sub-lethal effects on natural enemies (Desneux et al. 2007). Biopesticides are considered relatively benign to non-target species owing to their rapid breakdown, selectivity nature and reduced risk of insecticide resistance as plant extract, particularly crude extracts have multiple modes of action other than toxicity, such as repellency (Amoabeng et al. 2013; Dubey et al. 2011; Isman 2006; Koul et al. 2008; Tembo et al. 2018). Another important benefit of botanicals is that they tend to depend on "suites" of closely related active constituents rather than a single active ingredient; this diversity may delay or mitigate the development of resistance in pest populations to most botanicals (Koul 2004). Biopesticides have been used for centuries as means of managing pests until synthetic insecticides replaced plant extracts (Isman 1997). The interest in botanical insecticides is increasing but still accounts for less than $1 \%$ of crop protectants used globally (Isman 2008, 2017). In developing countries, plant extracts are often prepared from common weed species that grow around the field and obtained freely, with labour as the only cost, resulting in cheaper pest management option when compared with synthetic insecticides (Amoabeng et al. 2014; Isman 2017).

The field of botanical pesticides is highly active [see reviews by Boulogne et al. (2012), Isman and Grieneisen (2014), Isman (2017), Yang and Tang (1988)] but this review considers two novel aspects. First, we assess the extent to which the plant species used in conservation biological control studies have been the subject of research to determine if they have potentially useful biopesticidal properties. Second, we consider the practicalities of using plants that have dual use in promoting biological control and as sources of botanical pesticides (Fig. 1).

\section{Conservation biological control plant species}

The identification of plant species studied for habitat manipulation purposes began with the published review by Fiedler et al. (2008) and was followed by a search on the online ISI Web of Science database to from 1989 to 2018 using search terms: flower* AND "conservation biological control", flower* AND "natural enemy",and "habitat management" 


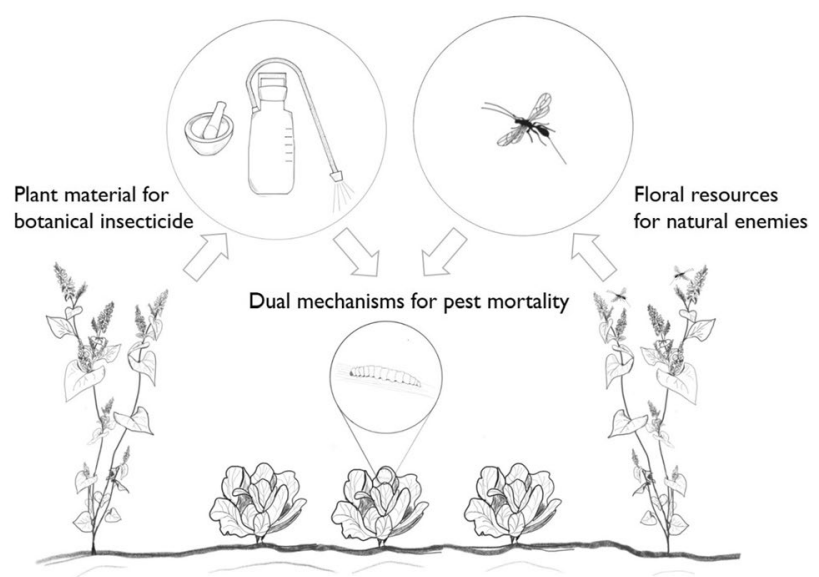

Fig. 1 A secondary plant species (such as buckwheat in this example) can potentially provide dual benefits for pest management, promoting biological control and providing insecticidal compounds to treat the crop

AND “conservation biological control". Between 1989 and 2006, 165 plant species belonging to 35 plant families were used in habitat manipulation studies. The criteria that were applied to the Fiedler et al. (2008) plant species included their effectiveness in previous habitat manipulation studies, frequency of natural enemy visitation, long flowering duration, availability of seeds, ease of establishment, agronomic suitability and the value of the plant as cover or alternative crop (Fiedler et al. 2008). The same search criteria were applied to conduct a follow-up database search (Table 1). Each plant species is listed once irrespective of the number of studies in which it was used. Where a plant was involved in more than one study, only one example reference is given.

Habitat manipulation studies from 1989 to 2006 involved 165 plant species belonging to 35 families and 188 new species from 29 families were the subject of work published between 2007 and 2018. The number of publications per year between 2007 and 2018 ranges from three (2009 and 2010) to ten in the first half of 2018 (Fig. 2). Conservation biological control studies are dominated by research from developed countries in North America and Europe as well as Australia, New Zealand and Japan (Wyckhuys et al. 2013). Tropical regions have more flowering species than temperate areas so there remains great potential of identifying additional plant species for use in habitat manipulation (Christenhusz and Byng 2016).

\section{Plant families used in habitat manipulation with insecticidal activity}

A search in Google, Google Scholar and Scopus using both the scientific and common names of each of the 283 plant species named in papers on conservation for the terms "'botanical insecticide", "plant extracts", biopesticides, "insecticidal activity" OR "pesticidal activity", revealed 15 (33.3\%) (Apiaceae, Apocynaceae, Asteraceae, Boraginaceae, Brassicaceae, Campanulaceae, Fabaceae, Lamiaceae, Myrtaceae, Papaveraceae, Polygonaceae, Primulaceae, Proteaceae, Rosaceae, Rubiaceae and Scrophulariaceae) out of 44 plant families that have been involved in habitat manipulation studies, had at least one plant genus or species with insecticidal activity. Proteaceae had one plant used in habitat manipulation in the same genus as another plant used as a biopesticide. All other plant families had at least one plant species tried for its insecticidal activity and tested in habitat manipulation studies.

Three families, Apiaceae, Asteraceae and Lamiaceae, had more than ten species with insecticidal activity and accounted for more than $70 \%$ of plant species studied. These families also had the highest number of species used in habitat manipulation studies. Of the 18 plant species in Apiaceae involved in habitat manipulation studies, 13 (72.2\%) have been tested and showed insecticidal activity. In the Asteraceae, 19 (25.7\%) out of the 74 plant species involved in habitat manipulation studies have been tested for their insecticidal activity. In addition, five more plants species in the same genus as some of the species for habitat manipulation studies have been used for their insecticidal activity. Lamiaceae had 11 (39.3\%) out of the 28 plants involved in habitat manipulation studies having insecticidal activity. Two families, Brassicaceae and Fabaceae, had several species involved in habitat manipulation studies but not many species in the families have been known to have insecticidal properties. Among the 14 species in the Brassicaceae family, five were identified to have activity against insects whilst two out of the 23 species in Fabaceae were identified to have activity against insects. Fagopyrum esculentum is one of the most studied species in habitat manipulation programs (Fiedler et al. 2008; Lavandero et al. 2006; Vattala et al. 2006) and was the only species with insecticidal activity among 12 species in Polygonaceae. At 2,500 ppm, a methanol extract of the grains of buckwheat was potent against the green peach aphid Myzus persicae (Sulzer) (Hemiptera: Aphidiade) (Lee and Rasmussen 2000).

Boulogne et al. (2012) reviewed plant families and species with insecticidal activity and found that 656 plant species belonging to 110 families are known to have insecticidal activity in which Lamiaceae alone had 181 (28\%) followed by Fabaceae, Asteraceae and Apiaceae. In all, 30 (66.8\%) out of the 44 plant families in this review do not have insecticidal activity. It is, however, possible that plant species in these families have not yet been studied enough for their activity against insects. 
Table 1 "Gap identification" of plants researched for conservation biological control and that have yet to be the subject of work to identify scope as sources of botanical insecticides (denoted by 'o') and plants that constitute "proof of concept" in having insecticidal proper- ties as well as utility in conservation biological control (denoted by ' + '; or more tentatively by a ' $x$ ' denoting a plant in the same genus showed insecticidal activity)

\begin{tabular}{|c|c|c|c|c|c|}
\hline Scientific name & Common name & $\begin{array}{l}\text { Botanical } \\
\text { insecticide } \\
\text { activity }\end{array}$ & Order of insects controlled & $\begin{array}{l}\text { Ref as } \\
\text { botanical } \\
\text { species }\end{array}$ & $\begin{array}{l}\text { Ref as } \\
\text { HM } \\
\text { species }\end{array}$ \\
\hline \multicolumn{6}{|l|}{ Apiaceae } \\
\hline Ammi majus $\mathrm{L}$. & Bishop's flower & + & Lepidoptera & 1 & $*$ \\
\hline Ammi visnaga (L.) Lamarck & Toothpick ammi & + & Diptera & 2,3 & $*$ \\
\hline Anethum graveolens L. & Dill & + & $\begin{array}{l}\text { Coleoptera, Blattodea, Diptera, } \\
\text { Lepidoptera }\end{array}$ & $4,5,6$ & $*$ \\
\hline Angelica atropurpurea $\mathrm{L}$. & Angelica & o & & & $*$ \\
\hline Anthriscus cerefolium (L.) & Chervil & + & Coleoptera, Diptera & 7 & $*$ \\
\hline $\begin{array}{l}\text { Anthriscus sylvestris (L.) Hoff- } \\
\text { mann }\end{array}$ & Cow parsley & + & Lepidoptera, Diptera & 63 & $*$ \\
\hline Apium graveolens $\mathrm{L}$. & Celery & + & Diptera & 8 & $*$ \\
\hline Carumcarvi L. & Caraway & + & Coleoptera & 9 & $*$ \\
\hline Conium maculatum & Poison hemlock & + & $\begin{array}{l}\text { Thysanoptera, Trombidiformes, } \\
\text { Hemiptera }\end{array}$ & 10,11 & 10 \\
\hline Conopodium majus & Pignut & o & & & 13 \\
\hline Coriandrum sativum L. & Coriander/cilantro & + & Coleoptera & 12,13 & 13 \\
\hline Daucus carota $\mathrm{L}$. & Wild carrot & + & Diptera, Coleoptera & 14,15 & $*$ \\
\hline Foeniculum vulgare Mill. & Fennel & + & Coleoptera & $12,16,17$ & $*$ \\
\hline Heracleum sphondylium L. & Eltrot & o & & & $*$ \\
\hline Heracleum maximum Bartr. & Cow parsnip & $\mathrm{x}$ & Coleoptera & 18 & $*$ \\
\hline Pastinaca sativa $\mathrm{L}$. & Wild parsnip & + & Lepidoptera & 19 & 2 \\
\hline Pimpinella anisum & Aniseed & + & Diptera & 20,21 & 10 \\
\hline Zizia aurea (L.) Koch & Golden alexanders & o & & & $*$ \\
\hline \multicolumn{6}{|l|}{ Apocynaceae } \\
\hline Apocynum cannabinum $\mathrm{L}$. & Indian hemp & o & & & $*$ \\
\hline Asclepsia syriaca & Common milkweed & o & & & 22 \\
\hline Asclepias tuberosa & Butterfly weed & o & & & 22 \\
\hline Asclepias fascicularis Dcne. & Milkweed & o & & & $*$ \\
\hline Asclepias incarnata $\mathrm{L}$. & Swamp milkweed & o & & & $*$ \\
\hline Asclepias syriaca $\mathrm{L}$. & Common milkweed & o & & & $*$ \\
\hline Asclepias tuberosa $\mathrm{L}$. & Butterfly weed & o & & & $*$ \\
\hline \multicolumn{6}{|l|}{ Asparagaceae } \\
\hline Asparagus acutifolius & Wild asparagus & o & & & 13 \\
\hline \multicolumn{6}{|l|}{ Asteraceae } \\
\hline Achillea millefolium $\mathrm{L}$. & Yarrow & + & Lepidoptera, Coleoptera & 22,24 & $*$ \\
\hline Achillea spp. & Yarrow & + & Lepidoptera, Coleoptera & 23,24 & $*$ \\
\hline Ageratina aromatic & Lesser snakeroot & $\mathrm{x}$ & Diptera & 25 & 14 \\
\hline Andryala integrifolia & Common andryala & o & & & 11 \\
\hline Anthemis arvensis $\mathrm{L}$. & Corn chamomile & $\mathrm{x}$ & Lepidoptera & 26 & $*$ \\
\hline Artemisia ludoviciana & Louisiana wormwood & + & Hemiptera, Orthoptera & 27,28 & 16 \\
\hline Aster nova-angliae $\mathrm{L}$. & Smooth aster & $\mathrm{x}$ & Lepidoptera & 26 & $*$ \\
\hline Aster novi-belgii $\mathrm{L}$. & New England aster & + & Lepidoptera & 26 & $*$ \\
\hline Baccharis pilularis DC. & New York aster & o & & & $*$ \\
\hline Baccharis viminea DC. & Coyotebrush & o & & & $*$ \\
\hline Cacalia atriplicifolia L. H. Rob. & Mule fat & o & & & $*$ \\
\hline Calendula arvensis & Field marigold & $\mathrm{x}$ & Lepidoptera & 29 & 7 \\
\hline Calendula officinalis L. & Calendula & + & Hemiptera & 30 & $*$ \\
\hline
\end{tabular}


Table 1 (continued)

\begin{tabular}{|c|c|c|c|c|c|}
\hline Scientific name & Common name & $\begin{array}{l}\text { Botanical } \\
\text { insecticide } \\
\text { activity }\end{array}$ & Order of insects controlled & $\begin{array}{l}\text { Ref as } \\
\text { botanical } \\
\text { species }\end{array}$ & $\begin{array}{l}\text { Ref as } \\
\text { HM } \\
\text { species }\end{array}$ \\
\hline Centaurea cyanus $\mathrm{L}$. & Garden cornflower & + & Hemiptera & 30 & $*$ \\
\hline Centaurea jacea $\mathrm{L}$. & Brownray knapweed & + & Hemiptera & 30 & $*$ \\
\hline Centaurea montana $\mathrm{L}$. & Mountain bluet & $\mathrm{x}$ & Hemiptera & 30 & $*$ \\
\hline Chondrilla juncea & Devil's grass & + & Coleoptera & 64 & 13 \\
\hline $\begin{array}{l}\text { Chrysanthemum maximum x } \\
\text { superbum cv. Snow Lady }\end{array}$ & Max chrysanthemum & o & & & $*$ \\
\hline $\begin{array}{l}\text { Chrysanthemum segetum (L.) } \\
\text { Fourr. }\end{array}$ & Corn marigold & o & & & $*$ \\
\hline Cichorium intybus $\mathrm{L}$. & Chickory & + & Diptera & 31 & $*$ \\
\hline Conoclinium coelestinum & Blue mistflower & o & & & 14 \\
\hline Coreopsis lanceolata $\mathrm{L}$. & Sand coreopsis & o & & & $*$ \\
\hline Coreopsis tinctoria Nutt. & Golden tickseed & o & & & $*$ \\
\hline Coreopsis verticillata L.b & Coreopsis & o & & & $*$ \\
\hline Cosmos bipinnatus & Garden cosmos & - & Diptera (less efficacious) & a & 9 \\
\hline Cosmos sulphureus & Yellow cosmos & o & & & 18 \\
\hline Crepis biennis & Rough hawksbeard & $\mathrm{o}$ & & & 12 \\
\hline Crepis capillaris & Smooth hawksbeard & o & & & 11 \\
\hline Dittrichia viscosa & False yellowhead & + & Hemiptera & 32 & 13 \\
\hline Echinacea pallida & Pale purple coneflower & - & Lepidoptera & $\mathrm{b}$ & 16 \\
\hline Echinacea purpurea (L.) Moench & Purple coneflower & + & Lepidoptera & 23 & $*$ \\
\hline Erigeron speciosus & Garden fleabane & o & & & 1 \\
\hline Eupatorium hyssopifolium & Hyssopleaf thoroughwort & + & Hemiptera, Diptera & 33 & 22 \\
\hline Eupatorium perfoliatum L. & Boneset & o & & & $*$ \\
\hline Gaillardia aristata Pursh & Common gaillardia & o & & & $*$ \\
\hline Gaillardia pulchella Foug. & Firewheel & o & & & $*$ \\
\hline Gazania rigens (L.) Gaertn. & Treasure-flower & o & & & $*$ \\
\hline Helianthus annus $\mathrm{L}$. & Sunflower & + & Isoptera, Coleoptera & 34,35 & $*$ \\
\hline Helianthus petiolaris & Lesser sunflower & o & & & 1 \\
\hline Helianthus strumosus $\mathrm{L}$. & Pale-leaved sunflower & o & & & $*$ \\
\hline $\begin{array}{l}\text { Helichrysum bracteatum (Vent.) } \\
\text { Andr. }\end{array}$ & Bracted strawflower & + & & 65 & $*$ \\
\hline Heliopsis helianthoides & False sunflower & o & & & 16 \\
\hline Heterotheca grandiflora & Telegraphweed & o & & & 1 \\
\hline Hypochaeris radicata & Catsear, flatweed & o & & & 12 \\
\hline $\begin{array}{l}\text { Layia platyglossa (Fisch. \& C.A. } \\
\text { Mey.) Gray }\end{array}$ & Tidy tips & o & & & $*$ \\
\hline Leontodon hispidus & Rough hawkbit & o & & & 12 \\
\hline Leucanthemum vulgare Lam. & Oxeye daisy & o & & & $*$ \\
\hline Leucanthemum $x$ superbum & Shasta daisy & o & & & $*$ \\
\hline Liatris aspera Michx. & Rough blazing star & o & & & $*$ \\
\hline Matricaria chamomilla & Scented mayweed & + & Coleoptera & 36 & 17 \\
\hline Matricaria recutita & Camomile & o & & & 9 \\
\hline Melampodium paludosum $\mathrm{L}$. & Medallion flower & o & & & $*$ \\
\hline Oligoneuron rigidum & & o & & & 16 \\
\hline Pityopsis graminifolia & Narrowleafsilgrass & o & & & 14 \\
\hline $\begin{array}{l}\text { Ratibidacolumnifera (Nutt.) Woot. } \\
\text { \&Standl. }\end{array}$ & Prairie coneflower & o & & & $*$ \\
\hline Ratibida pinnata (Vent.) Barnh. & Yellow coneflower & o & & & $*$ \\
\hline Rudbeckia hirta L. & Blackeyed Susan & + & & 66 & $*$ \\
\hline
\end{tabular}


Table 1 (continued)

\begin{tabular}{|c|c|c|c|c|c|}
\hline Scientific name & Common name & $\begin{array}{l}\text { Botanical } \\
\text { insecticide } \\
\text { activity }\end{array}$ & Order of insects controlled & $\begin{array}{l}\text { Ref as } \\
\text { botanical } \\
\text { species }\end{array}$ & $\begin{array}{l}\text { Ref as } \\
\text { HM } \\
\text { species }\end{array}$ \\
\hline Senecio obovatus Muhl. exWilld. & Round-leaved ragwort & o & & & $*$ \\
\hline Senecio vulgaris & Old-man-in-the-Spring & - & Coleoptera & $\mathrm{c}$ & 7 \\
\hline Silphium perfoliatum $\mathrm{L}$. & Cup plant & - & Diptera & $\mathrm{d}$ & $*$ \\
\hline Silybum marianum & Saint Mary's thistle & o & & & 17 \\
\hline Solidago canadensis L. & Late goldenrod & + & Diptera & 67 & $*$ \\
\hline Solidago juncea & Early goldenrod & o & & & 20 \\
\hline Solidago riddellii Frank ex Riddell & Riddell's goldenrod & o & & & $*$ \\
\hline Solidago speciosa Nutt. & Showy goldenrod & o & & & $*$ \\
\hline Solidigao spp & Goldenrod & o & & & $*$ \\
\hline Symphyotrichum shortii & Short's aster & o & & & 16 \\
\hline Tagetes erecta & Aztec marigold & + & Coleoptera & 15 & 4 \\
\hline Tagetes panda & & o & & & 2 \\
\hline Tagetes patula $\mathrm{L}$. & French marigold & + & Diptera & 37 & $*$ \\
\hline Tanacetum vulgare $\mathrm{L}$. & Common tansy & + & Lepidoptera & 68 & $*$ \\
\hline Vernonia missurica Raf. & Ironweed & o & & & * \\
\hline Zinnia elegans Jacquin & Common zinnia & - & Hemiptera & $\mathrm{e}$ & $*$ \\
\hline Zinnia hybrida & & o & & & 18 \\
\hline \multicolumn{6}{|l|}{ Boraginaceae } \\
\hline Borago officinalis $\mathrm{L}$. & Borage & + & Coleoptera & 38 & $*$ \\
\hline Echium lycopsis L. p.p. & Borage & $\mathrm{x}$ & & & $*$ \\
\hline Echium plantagineum & Purple viper's-bugloss & o & & & 13 \\
\hline Echium vulgare $\mathrm{L}$. & Common viper's bugloss & + & Lepidoptera & 39 & $*$ \\
\hline \multicolumn{6}{|l|}{ Brassicaceae } \\
\hline Aurinia saxitalis (L.) Desv. & Aurinia & o & & & $*$ \\
\hline Brassica juncea (L.) Czern. & Mustard & + & Coleoptera & 69 & $*$ \\
\hline Camelina sativa & Gold-of-pleasure & o & & & 8 \\
\hline Capsella bursa-pastoris & Shepherd's purse & o & & & 13 \\
\hline Diplotaxis tenuifolia & Perennial wall-rocket & o & & & 17 \\
\hline Hirschfeldia incana & Shortpod mustard & o & & & 17 \\
\hline Iberis sp. & Candytuft & o & & & $*$ \\
\hline Lobularia maritima (L.) Desv. & Alyssum & o & & & $*$ \\
\hline Moricandia sp & Violet cabbage & o & & & 19 \\
\hline Nasturtium sp. & & o & & & $*$ \\
\hline Raphanus sativus L. & Daikon/radish & + & Diptera & 40 & $*$ \\
\hline Raphanus raphanistrum & White charlock & + & Coleoptera & 41 & 13 \\
\hline Sinapis alba $\mathrm{L}$. & White mustard & + & Diptera, Lepidoptera & 40,42 & $*$ \\
\hline Sinapis arvensis & Wild mustard & + & Lepidoptera & 42 & 8 \\
\hline \multicolumn{6}{|l|}{ Campanulaceae } \\
\hline Campanula glomerata $\mathrm{L}$. & Bell flower & o & & & $*$ \\
\hline Campanula persicifolia $\mathrm{L}$. & Peach-leaved bellflower & o & & & $*$ \\
\hline Campanula rotundifolia & Harebell & - & Lepidoptera & $\mathrm{f}$ & 1 \\
\hline Jasione montana & Sheep's bit scabious & o & & & 11 \\
\hline Lobelia cardinalis $\mathrm{L}$. & Cardinal flower & o & & & $*$ \\
\hline Lobelia siphilitica $\mathrm{L}$. & Great blue lobelia & + & Diptera & 43 & $*$ \\
\hline \multicolumn{6}{|l|}{ Caprifoliaceae } \\
\hline Knautia arvensis & Field scabious & o & & & 12 \\
\hline Lonicera etrusca & Etruscan honeysuckle & o & & & 7 \\
\hline
\end{tabular}


Table 1 (continued)

\begin{tabular}{|c|c|c|c|c|c|}
\hline Scientific name & Common name & $\begin{array}{l}\text { Botanical } \\
\text { insecticide } \\
\text { activity }\end{array}$ & Order of insects controlled & $\begin{array}{l}\text { Ref as } \\
\text { botanical } \\
\text { species }\end{array}$ & $\begin{array}{l}\text { Ref as } \\
\text { HM } \\
\text { species }\end{array}$ \\
\hline $\begin{array}{l}\text { Sambucus mexicana K. Presl ex } \\
\text { DC. }\end{array}$ & Elderberry & o & & & $*$ \\
\hline Sambucus racemosa $\mathrm{L}$. & Red-berried elder & o & & & $*$ \\
\hline \multicolumn{6}{|l|}{ Caryophyllaceae } \\
\hline Agrostemma githago & Common corncockle & o & & & $*$ \\
\hline Gypsophila sp. & Gypsophila & o & & & $*$ \\
\hline Gypsophila elegans & Showy baby's-breath & o & & & 9 \\
\hline Silene alba (P. Mill.) Krause & Bladder campion & - & Hemiptera & $\mathrm{e}$ & $*$ \\
\hline Silenegallica & Common catchfly & o & & & 13 \\
\hline Spergula arvensis & Corn spurry & o & & & 13 \\
\hline Stellaria media & Chickweed & o & & & 7 \\
\hline \multicolumn{6}{|l|}{ Chenopodiaceae } \\
\hline Chenopodium quinoa Willd. & Quinoa & o & & & $*$ \\
\hline \multicolumn{6}{|l|}{ Euphorbiaceae } \\
\hline Euphorbia epithymoides L. & Flowering spurge & o & & & $*$ \\
\hline \multicolumn{6}{|l|}{ Fabaceae } \\
\hline Amorphacanescens Pursh & Leadplant & $\mathrm{x}$ & Diptera & 44 & $*$ \\
\hline Cassia fasciculata Michx. & Partridge pea & o & & & $*$ \\
\hline Crotalaria juncea & Sun hemp & + & Coleoptera & 45 & 6 \\
\hline Dalea purpurea & Purple prairie clover & o & & & 16 \\
\hline Desmodium canadense (L.) DC. & Showy tick trefoil & o & & & $*$ \\
\hline Lespedeza hirta (L.) Hornem. & Hairy bush-clover & o & & & $*$ \\
\hline Lotus corniculatus $\mathrm{L}$. & Bird's foot trefoil & o & & & $*$ \\
\hline Lupinus albus L. & White lupine & o & & & $*$ \\
\hline Medicago lupulina & Black medick & o & & & 12 \\
\hline Medicago sativa $\mathrm{L}$. & Alfalfa & o & & & $*$ \\
\hline Lotus australis & Austral trefoil & o & - & & 3 \\
\hline Onobrychis viciifolia & Common sainfoin & o & & & 1 \\
\hline Ononis natrix & Shrubby rest-harrow & o & & & 2 \\
\hline Phaseolus vulgaris & Common bean & o & & & 15 \\
\hline Trifolium hybridum & Alsike clover & $\mathrm{o}$ & & & 2 \\
\hline Trifolium incarnatum $\mathrm{L}$. & Crimson clover & o & & & $*$ \\
\hline Trifolium pratense & Red clover & o & & & 2 \\
\hline Trifolium repens $\mathrm{L}$. & Ladino, white clover & o & & & $*$ \\
\hline Trifolium subterrraneum L. & Subclover & o & & & $*$ \\
\hline Vicia cracca & Tufted vetch & o & & & 2 \\
\hline Vicia faba $\mathrm{L}$. & Faba bean & + & Hemiptera & 46 & $*$ \\
\hline Vicia sativa $\mathrm{L}$. & Common vetch & o & & & $*$ \\
\hline Vicia villosa & Hairy vetch & o & & & 10 \\
\hline \multicolumn{6}{|l|}{ Gentianaceae } \\
\hline Exacum sp. & Exacum & o & & & $*$ \\
\hline Geranium endressii Gayd & Cranesbill & o & & & * \\
\hline Geranium maculatum $\mathrm{L}$. & Wild geranium & $\mathrm{o}$ & & & $*$ \\
\hline Geranium pyrenaicum & Hedgerow cranesbill & o & & & 12 \\
\hline \multicolumn{6}{|l|}{ Hydrophyllaceae } \\
\hline Hydrophyllum virginianum $\mathrm{L}$. & Virginia waterleaf & o & & & $*$ \\
\hline Nemophilamenziesii Hook. \& Arn. & Baby blue eyes & o & & & $*$ \\
\hline Phacelia campanularia Gray & Wild Canterbury bells & o & & & $*$ \\
\hline
\end{tabular}


Table 1 (continued)

\begin{tabular}{|c|c|c|c|c|c|}
\hline Scientific name & Common name & $\begin{array}{l}\text { Botanical } \\
\text { insecticide } \\
\text { activity }\end{array}$ & Order of insects controlled & $\begin{array}{l}\text { Ref as } \\
\text { botanical } \\
\text { species }\end{array}$ & $\begin{array}{l}\text { Ref as } \\
\text { HM } \\
\text { species }\end{array}$ \\
\hline Phacelia tanacetifolia Benth. & Phacelia & o & & & $*$ \\
\hline \multicolumn{6}{|l|}{ Hypericaceae } \\
\hline Hypericum perforatum & Common Saint John's wort & o & & & 13 \\
\hline \multicolumn{6}{|l|}{ Iridaceae } \\
\hline Iris germinica $\mathrm{L}$. & Iris & $\mathrm{o}$ & & & $*$ \\
\hline \multicolumn{6}{|l|}{ Lamiaceae } \\
\hline Acinos arvensis Dandy & Basil thyme & $\mathrm{o}$ & & & $*$ \\
\hline Agastache foeniculum (Pursh) & Anise hyssop & + & Diptera & 2 & $*$ \\
\hline Agastache nepetoides (L.) Kuntze & Yellow giant hyssop & $\mathrm{o}$ & & & $*$ \\
\hline $\begin{array}{l}\text { Agastache rugose Fisch. } \\
\text { \&C.A.Mey.) }\end{array}$ & Korean licorice mint & - & Coleoptera & $\mathrm{g}$ & * \\
\hline Buddleja davidii & Orange eye & o & & & 14 \\
\hline Calamintha baetica Mill. & Woodland calamint & o & & & 13 \\
\hline Calamintha nepeta & Lesser calamint & + & Diptera & 47 & 14 \\
\hline Clinopodium vulgare & Wild basil & o & & & 2 \\
\hline Cordia verbanacea(Jacq.) & Cordia & - & Coleoptera & $\mathrm{h}$ & 5 \\
\hline Lamium purpureum $L$. & Red dead-nettle & o & & & 7 \\
\hline Lavandula stoechas L. & French lavender & + & Diptera, Coleoptera & 2,48 & 13 \\
\hline Mellisa officinalis L. & Common balm & + & Lepidoptera & 49 & 5 \\
\hline Mentha piperita $\mathrm{L}$. & Peppermint & + & Coleoptera, Diptera & 50 & 5 \\
\hline Mentha satureioides $\mathrm{R} . \mathrm{Br}$. & Bushmint & + & Lepidoptera, Hemiptera & 51 & 3 \\
\hline Mentha spicata $\mathrm{L}$. & Spearmint & + & Diptera & 52 & $*$ \\
\hline Monarda citriodora Cerv. ex Lag. & Lemon bee balm & o & & & $*$ \\
\hline Monarda fistulosaL. & Bee balm & + & Trombidiformes & 53 & 20 \\
\hline Monarda punctate $\mathrm{L}$. & Horsemint & o & & & $*$ \\
\hline Ocimum basilicum L. & Basil & + & Coleoptera, Lepidoptera, Hemiptera & 17,54 & 5 \\
\hline Origanum vulgare $\mathrm{L}$. & Wild marjoram & + & Trombidiformes, Lepidoptera & 53,62 & 12 \\
\hline Prunella vulgaris L. & Selfheal & o & & & $*$ \\
\hline $\begin{array}{l}\text { Pycnanthemum tenuifolium } \\
\text { Schrad. }\end{array}$ & Common horsemint & o & & & 22 \\
\hline Rosmarinus officinalis $L$. & Rosemary & + & Coleoptera & 12,55 & 7 \\
\hline Salvia farinacea Benth. & Mealy-cup sage & $\mathrm{o}$ & & & $*$ \\
\hline Salvia uliginosa Benth. & Bog sage & o & & & $*$ \\
\hline Salvia viridis $\mathrm{L}$. & Clary sage & o & & & $*$ \\
\hline Scutellaria integrifolia $\mathrm{L}$. & Helmet flower & o & & & 22 \\
\hline $\begin{array}{l}\text { Westringia fruticose (Willd.) } \\
\text { Druce }\end{array}$ & Coastal rosemary & o & & & 3 \\
\hline \multicolumn{6}{|l|}{ Liliaceae } \\
\hline Allium cernuum Roth & Nodding wild onion & o & & & $*$ \\
\hline Convallaria majalis L. & Lily of the valley & o & & & $*$ \\
\hline \multicolumn{6}{|l|}{ Linaceae } \\
\hline Linum grandiflorum Desf. & Scarlet fla & o & & & $*$ \\
\hline \multicolumn{6}{|l|}{ Lythraceae } \\
\hline Lythrum salicariaL. & Purple loosestrife & o & & & 12 \\
\hline \multicolumn{6}{|l|}{ Malvaceae } \\
\hline Malva moschate L. & Musk mallow & o & & & 12 \\
\hline Malva neglectaWallr. & Common mallow & o & & & 13 \\
\hline Malva sylvestris $L$. & High mallow & $\mathrm{o}$ & & & 7 \\
\hline
\end{tabular}


Table 1 (continued)

\begin{tabular}{|c|c|c|c|c|c|}
\hline Scientific name & Common name & $\begin{array}{l}\text { Botanical } \\
\text { insecticide } \\
\text { activity }\end{array}$ & Order of insects controlled & $\begin{array}{l}\text { Ref as } \\
\text { botanical } \\
\text { species }\end{array}$ & $\begin{array}{l}\text { Ref as } \\
\text { HM } \\
\text { species }\end{array}$ \\
\hline \multicolumn{6}{|l|}{ Myrtaceae } \\
\hline Callistemom citrinus (Curtis) & Crimson/common red & + & Coleoptera & 56 & 3 \\
\hline Leptospermum cv Rudolpd & Tea tree & o & & & 3 \\
\hline \multicolumn{6}{|l|}{ Onagraceae } \\
\hline $\begin{array}{l}\text { Clarkia amoena (Lehm.) A. Nels. } \\
\text { \& J.F. Macbr. }\end{array}$ & Farewell-to-spring & o & & & * \\
\hline $\begin{array}{l}\text { Clarkia concinna (Fisch. \& C.A. } \\
\text { Mey.) Greene }\end{array}$ & Red ribbons & o & & & * \\
\hline Clarkia unquiculata Lindl. & Elegant clarkia & o & & & * \\
\hline Oenothera speciosa Nutt. & Pinkladies & o & & & * \\
\hline Oenotherabiennis L. & Evening primrose & o & & & $*$ \\
\hline \multicolumn{6}{|l|}{ Paeoniaceae } \\
\hline Paeonia lactiflora Pallas & Peony & $\mathrm{o}$ & & & $*$ \\
\hline \multicolumn{6}{|l|}{ Papaveraceae } \\
\hline Eschscholzia californica Cham. & California poppy- & o & & & * \\
\hline Papaver rhoeas $\mathrm{L}$. & Common poppy & o & & & $*$ \\
\hline Papaver somniferum & Opium poppy & + & Isoptera & 57 & 17 \\
\hline $\begin{array}{l}\text { Stylomecon heterophylla (Benth.) } \\
\text { G. Taylor }\end{array}$ & Wind poppy & o & & & $*$ \\
\hline \multicolumn{6}{|l|}{ Plantaginaceae } \\
\hline Penstemon digitalisNutt. ex Sims & foxglove beard-tongue & o & & & 1 \\
\hline Veronicastrum virginacum & & o & & & 16 \\
\hline Veronica persicaPoir. & Common field-speedwell & o & & & 7 \\
\hline \multicolumn{6}{|l|}{ Poaceae } \\
\hline Agrostis capillaries L. & Colonial bent & o & & & 2 \\
\hline Andropogon gerardi Vitman & Big bluestem & o & & & 16 \\
\hline $\begin{array}{l}\text { Bouteloua curtipendula (Michx.) } \\
\text { Torr. }\end{array}$ & Side oats grama & o & & & 16 \\
\hline Cynosurus cristatus L. & Crested dog's-tail & o & & & 2 \\
\hline Elymus Canadensis L. & Canada wild rye & o & & & 16 \\
\hline Festuca rubraL. & Red fescue & o & & & 2 \\
\hline Panicum virgatum $L$. & Switchgrass & o & & & 16 \\
\hline Poa pratensis $\mathrm{L}$. & Kentucky bluegrass & o & & & 2 \\
\hline $\begin{array}{l}\text { Schizachyriums coparium (Michx.) } \\
\text { Nash }\end{array}$ & Little bluestem & o & & & 22 \\
\hline Sorghastrum nutans (L.) Nash & Yellow Indiangrass & o & & & 16 \\
\hline $\begin{array}{l}\text { Sporobolus clandestinus (Biehler) } \\
\text { A.S. Hitchc. }\end{array}$ & Rough dropseed & o & & & 16 \\
\hline \multicolumn{6}{|l|}{ Polemoniaceae } \\
\hline Gilia tri-color Benth. & Bird's eyes & o & & & * \\
\hline $\begin{array}{l}\text { Linanthus grandiflorus (Benth.) } \\
\text { Greene }\end{array}$ & Mountain phlox & o & & & $*$ \\
\hline \multicolumn{6}{|l|}{ Polygonaceae } \\
\hline Eriogonum compositum & Arrowleaf buckwheat & o & & & 14 \\
\hline Eriogonum douglasii & Douglas' buckwheat & o & & & 14 \\
\hline $\begin{array}{l}\text { Eriogonum elatum Dougl. } \\
\text { exBenth. }\end{array}$ & Tall woolly buckwheat & o & & & 14 \\
\hline Eriogonum fasciculatum Benth. & California buckwheat & o & & & $*$ \\
\hline Eriogonum giganteum $\mathrm{S}$. Wats. & St Catherine's lace & o & & & \\
\hline
\end{tabular}


Table 1 (continued)

\begin{tabular}{|c|c|c|c|c|c|}
\hline Scientific name & Common name & $\begin{array}{l}\text { Botanical } \\
\text { insecticide } \\
\text { activity }\end{array}$ & Order of insects controlled & $\begin{array}{l}\text { Ref as } \\
\text { botanical } \\
\text { species }\end{array}$ & $\begin{array}{l}\text { Ref as } \\
\text { HM } \\
\text { species }\end{array}$ \\
\hline Eriogonum heracleoides & Parsnipflower buckwheat & o & & & 14 \\
\hline Eriogonum microthecum & Slender buckwheat & o & & & 14 \\
\hline Eriogonum niveum & Snow buckwheat & o & & & 14 \\
\hline Eriogonum sphaerocephalum & Rock buckwheat & o & & & 14 \\
\hline Eriogonum strictum & Blue Mountain buckwheat & o & & & 14 \\
\hline Eriogonum thymoides & Thymeleaf buckwheat & o & & & 14 \\
\hline Fagopyrum esculentum Moench & Buckwheat & + & Hemiptera & 58 & $*$ \\
\hline \multicolumn{6}{|l|}{ Primulaceae } \\
\hline Primula veris $\mathrm{L}$. & Cowslip & + & Coleoptera & 59 & 2 \\
\hline \multicolumn{6}{|l|}{ Proteaceae } \\
\hline Grevillea cv Bronze Rambler & & $\mathrm{x}$ & & & 3 \\
\hline \multicolumn{6}{|l|}{ Ranunculaceae } \\
\hline Anemone canadensis $\mathrm{L}$. & Canada anemone & o & & & $*$ \\
\hline Aquilegia canadensis $\mathrm{L}$. & Columbine & o & & & $*$ \\
\hline $\begin{array}{l}\text { Consolidaambigua (L.) P.W. Ball } \\
\text { \& Heywood }\end{array}$ & Doubtful knight's-spur & o & & & * \\
\hline Ranunculus ollissiponensis Pers & & o & & & 7 \\
\hline Thalictrum aquilegifolium $\mathrm{L}$. & Meadow rue & o & & & $*$ \\
\hline \multicolumn{6}{|l|}{ Rhamnaceae } \\
\hline Ceanothus sp. & California lilac & o & & & $*$ \\
\hline Ceanothus americanus $\mathrm{L}$. & New Jersey tea & o & & & $*$ \\
\hline Rhamnus californica Eschsch. & Coffeeberry & o & & & $*$ \\
\hline \multicolumn{6}{|l|}{ Rosaceae } \\
\hline Fragaria virginiana Duchesne & Wild strawberry & o & & & $*$ \\
\hline Heteromeles arbutifolia & Toyon & o & & & $*$ \\
\hline Potentialla fruticoseauct. non L. & Shrubby cinquefoil & o & & & $*$ \\
\hline $\begin{array}{l}\text { Prunusilicifolia Nutt. ex Hook. } \\
\text { \& Arn. }\end{array}$ & Hollyleaf cherry & o & & & $*$ \\
\hline Quillaja saponaria Molina & Soapbark tree & + & Diptera & 60 & $*$ \\
\hline Rosa setigeraMichx. & Michigan rose & o & & & $*$ \\
\hline Spiraea alba Duroi & Meadowsweet & o & & & $*$ \\
\hline \multicolumn{6}{|l|}{ Rubiaceae } \\
\hline Cephalanthus occidentalis $\mathrm{L}$. & Buttonbush & $\mathrm{o}$ & & & $*$ \\
\hline GaliumaparineL. & Cleavers & + & Lepidoptera & 61 & 14 \\
\hline Galiumverum L. & Yellow bedstraw & o & & & 12 \\
\hline \multicolumn{6}{|l|}{ Rutaceae } \\
\hline Ruta graveolens $\mathrm{L}$. & Rue & - & Coleoptera & $\mathrm{h}$ & $*$ \\
\hline \multicolumn{6}{|l|}{ Salicacaeae } \\
\hline Salix sp. & Willow & o & & & * \\
\hline \multicolumn{6}{|l|}{ Saxifragaceae } \\
\hline Astilbe $x$ arendsii & Bridal veil & o & & & $*$ \\
\hline Heuchera americana $\mathrm{L}$. & Alum root & o & & & $*$ \\
\hline \multicolumn{6}{|l|}{ Scrophulariaceae } \\
\hline $\begin{array}{l}\text { Collinsia heterophylla Buist ex } \\
\text { Graham }\end{array}$ & Chinese houses & o & & & $*$ \\
\hline Linaria maroccana Hook. f. & Toadflax & o & & & $*$ \\
\hline Myoporum parvifoliumR.Br. & Creeping boobialla & o & & & 3 \\
\hline Linaria saxatilisL. & & + & Coleoptera & 16 & 13 \\
\hline Penstemon hirsutus (L.) Willd. & Penstemon & o & & & $*$ \\
\hline
\end{tabular}


Table 1 (continued)

\begin{tabular}{|c|c|c|c|c|c|}
\hline Scientific name & Common name & $\begin{array}{l}\text { Botanical } \\
\text { insecticide } \\
\text { activity }\end{array}$ & Order of insects controlled & $\begin{array}{l}\text { Ref as } \\
\text { botanical } \\
\text { species }\end{array}$ & $\begin{array}{l}\text { Ref as } \\
\text { HM } \\
\text { species }\end{array}$ \\
\hline Scrophularia marilandica $\mathrm{L}$. & Late figwor & o & & & $*$ \\
\hline Verbascum phlomoides L. & Orange mullein & o & & & $*$ \\
\hline \multicolumn{6}{|l|}{ Solanaceae } \\
\hline $\begin{array}{l}\text { Nicotiana alata } \times \text { sanderae Link } \\
\quad \& \text { Otto }\end{array}$ & Flowering tobacco & o & & & $*$ \\
\hline Petunia $\times$ hybridaVilmg & Petunia & o & & & $*$ \\
\hline \multicolumn{6}{|l|}{ Tropaeolaceae } \\
\hline Tropaeolum majus L. & Nasturtium & o & & & $*$ \\
\hline \multicolumn{6}{|l|}{ Urticaceae } \\
\hline Urtica urens $\mathrm{L}$. & Dwarf nettle & o & & & 17 \\
\hline \multicolumn{6}{|l|}{ Verbenaceae } \\
\hline Aloysia virgate Paláu & Beebrushes & o & & & 14 \\
\hline Phyla nodiflora (L.) Greene & Frog fruit & o & & & 14 \\
\hline Verbena canadensis (L.) Brittonh & Rose verbena & o & & & * \\
\hline Verbena stricta Vent. & Hoary vervain & o & & & $*$ \\
\hline
\end{tabular}

Data set based on the literature published from 1989 to 2018; species with * are from Fiedler et al. (2008) review of conservation biological control

Plants involved in habitat manipulation from 1989 to 2018. Species with * are from Fiedler et al. (2008)

Reference for habitat manipulation plant species with * are found in Fiedler et al. (2008)

+ Insecticidal activity

- Plant species tested against insects but with less/no activity

O plant species not tested

$\mathrm{X}$ Different species in the same genus with insecticidal activity

References for plants for habitat manipulation: 1. (Pellissier and Jabbour 2018) 2. (Campbell et al. 2017) 3. (Pandey et al. 2018) 4. (Haro et al. 2018) 5. (Batista et al. 2017) 6. (Trisnawati and Azis 2017) 7. (Villa et al. 2016) 8. (Tschumi et al. 2016) 9. (van Rijn et al. 2016) 10. (van Rijn et al. 2016) 11. (Villa et al. 2016) 12. (Hatt et al. 2017) 13. (Nave et al. 2016) 14. (Sivinski 2014) 15. (Balzan et al. 2014) 16. (Gill et al. 2014) 17. (Martinez-Una et al. 2013) 18. (Gontijo et al. 2013) 19. (Diaz et al. 2012) 20. (Walton and Isaacs 2011) 21. (Tuell et al. 2008) 22. (Frank et al. 2008)

Reference as botanical insecticide. 1. (El-Ghar et al. 1996) 2.(Ebadollahi 2013) 3. (Pavela et al. 2016) 4. (Babri et al. 2012) 5. (Sousa et al. 2013) 6. (Sousa et al. 2015) 7. (Evergetis and Haroutounian 2014) 8. (Khater and Khater 2009) 9. (Lopez et al. 2008) 10. (Chermenskaya et al. 2010) 11. (Gokce et al. 2016) 12. (Pavela and Benelli 2016) 13. (Rani 2012) 14. (Momin and Nair 2002) 15. (Azad et al. 2013) 16. (Koul et al. 2008) 17. (Cosimi et al. 2009) 18. (Alkan et al. 2017) 19. (Berenbaum et al. 1991) 20. (Santos et al. 1998) 21.(Park et al. 2006) 22. (Hasheminia et al. 2011) 23. (Pavela 2010) 24. (Nenaah et al. 2015) 25. (Rajeswary and Govindarajan 2013) 26. (Kumar et al. 2017) 27. (Rizvi et al. 2018) 28. (Blust and Hopkins 1987) 29. (Medhini et al. 2012) 30. (Alexenizer and Dorn 2007) 31. (Mansour et al. 2014) 32. (Merah and Djazouli 2016) 33. (Tabanca et al. 2010) 34. (Aihetasham et al. 2017) 35. (Mullin et al. 1991) 36. (Padin et al. 2013) 37. (Macedo et al. 1997) 38. (Ahmed et al. 2015) 39. (Vukajlović et al. 2018) 40. (Khater and Khater 2009) 41. (Jbilou et al. 2008) 42. (Sivaraman et al. 2014) 43. (Pavela 2009) 44. (Liang et al. 2015) 45. (Hossain and Haque 2010) 46. (Meradsi and Laamari 2016) 47. (Guarrera 1999) 48. (Ebadollahi 2011) 49. (Pavela 2004) 50. (Kumar et al. 2011) 51. (Arnoabeng et al. 2018) 52. (Koul et al. 2008) 53. (Momen et al. 2014) 54. (Amoabeng et al. 2013) 55. (Regnault-Roger et al. 2004) 56. (Lee et al. 2004) 57. (Ahmed et al. 2011) 58. (Lee et al. 2000) 59. (Sibul et al. 2001) 60. (Pelah et al. 2002) 61. (Morimoto et al. 2002) 62. (Akhtar and Isman 2004) 63. (Kozawa et al. 1982) 64. (Boussaada et al. 2008) 65. (Yeom et al. 2015) 66. (Guillet et al. 1997 ) 67. (Raghavendra et al. 2013) 68. (Larocque et al. 1999) 69. (Kim et al. 2003)

Reference for tested plant but less efficacious/no activity: a. (Mohankumar et al. 2016) b. (Pavela 2010) c. (Pascual-Villalobos and Robledo 1999) d. (Pavela 2009) e. (Alexenizer and Dorn 2007) f. (Pavela 2011) g. (Kim et al. 2003) h. (Moreira et al. 2007)

\section{The Apiaceae}

The Apiaceae (umbellifers) is the 16th largest angiosperm family with 442 genera and 3575 species in which most of the aromatic flowering plants are found (Christenhusz and Byng 2016). It has a global distribution and many species with both habitat manipulation and insecticidal activity traits (Christenhusz and Byng 2016). Apiaceae species are annual, biennial or perennial herbs and woody shrubs and small trees that produce colorful inflorescences that secrete nectar attracting pollinators including bees, moths and beetles (Heywood et al. 2007). Their growth habit makes them agronomically suitable as habitat manipulation species (Fiedler et al. 2008). The flat headed morphology of their 
Fig. 2 Temporal trend in published papers on habitat manipulation between 2007 and July 2018. Previously published papers on habitat manipulation (1989 to July 2006 were the subject of Fiedler et al. (2008))

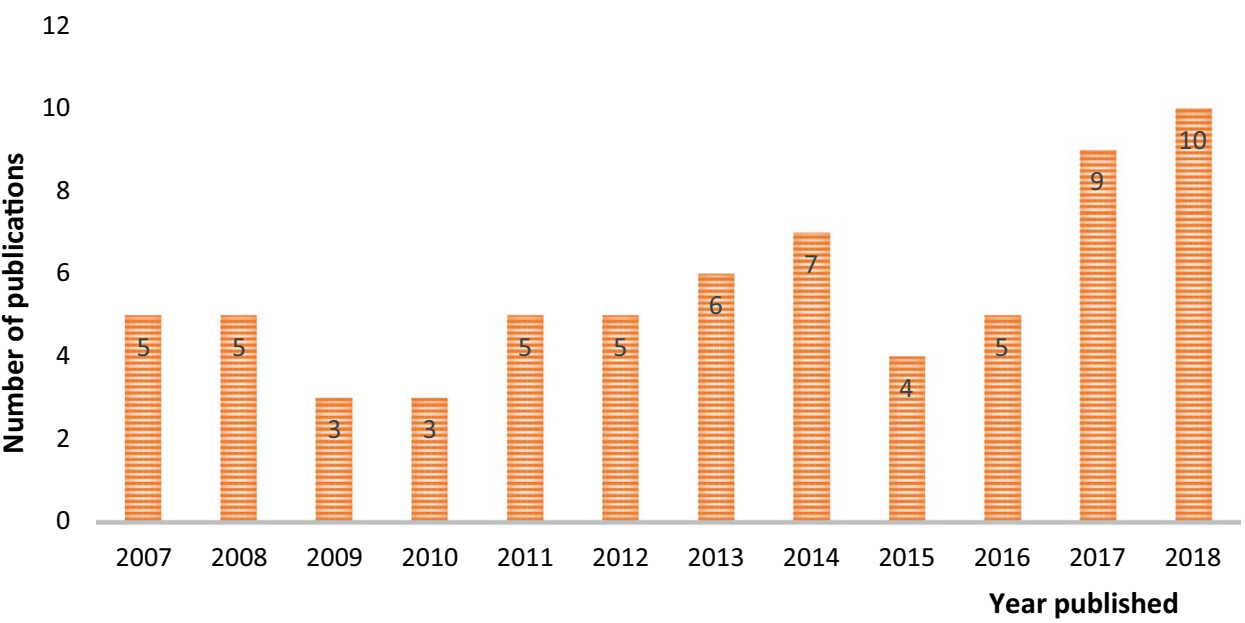

inflorescence provides easy landing and access to nectaries for natural enemies encouraging visitation (Heywood et al. 2007).

Plants in the Apiaceae produce secondary metabolites including coumarins, monoterpenes and sesquiterpenes (Lee and Rasmussen 2000). Essential oils have been tested as being acaricidal (Attia et al. 2011), bactericidal (Glisic et al. 2007; Matasyoh et al. 2009) and for medicinal purposes (Lee and Rasmussen 2000; Maulidiani et al. 2014). Essential oils from Apiaceae species have been used against stored product pests including the bean weevil Acanthoscelides obtectus Say (Coleoptera: Bruchidae) (Regnault-Roger et al. 1993), the cigarette beetle Lasioderma serricorne (F.) (Coleoptera: Anobiidae) and wheat flour beetle Tribolium castaneum Herbst (Coleoptera: Tenebrionidae) (Kim et al. 2003). Insecticidal activity against turnip aphids, Lipaphis pseudobrassicae (Davis) (Hemiptera: Aphididae), pea aphid, Acyrthosiphon pisum (Harris) (Hemiptera: Aphididae) and the green peach aphid, M. persicae has been reported (Dancewicz et al. 2012; Sampson et al. 2005).

\section{The Asteraceae (Compositae) family}

The Asteraceae (daisy) is the second largest plant family after Orchidaceae (Stevens and Davis 2001) with 24,700 species in 1623 genera and a worldwide distribution (Christenhusz and Byng 2016). Asteraceae species have clusters of inflorescence that appears to be a single flower often referred to as head (Schmid 2004). The entire flower head moves towards the direction of the sun and that maximizing reflectivity which may enhance the attraction of pollinators and other beneficial insects (Schmid 2004), which along with their growth habit makes them largely acceptable for habitat manipulation (Altieri et al. 2005). A larger number of plants in the family are herbaceous, with shrubs and trees rare (Okunade 2002). Species in the Asteraceae have been exploited for their insecticidal activity against crop and storage pests (Gbolade et al. 2011) and also against several pathogenic organisms with success (Del-Vechio-Vieira et al. 2009; Senatore et al. 2004). The chemical composition of some species including Ageratum conyzoides L. (Asteraceae) has been well described (Chu et al. 2010; de Souza et al. 2009; Nenaah et al. 2015; Okunade 2002). The insecticidal activity of an aqueous extract of $A$. conyzoides has shown success rates comparable to chemical insecticides against diamondback moth, Plutella xylostella L. (Lepidoptera: Plutellidae) (Amoabeng et al. 2013; Bhathal et al. 1994).

\section{The Lamiaceae}

The Lamiaceae (mints and deadnettles) is characterised by many aromatic species (Heywood et al. 2007).The family is composed of 7530 species in 241 genera and globally distributed (Christenhusz and Byng 2016). The Lamiaceae includes trees, shrubs, subshrubs and herbs that are annuals or perennials (Harley et al. 2004). The Lamiaceae has a large variety of species composed of plants that may bloom early in the season (annuals) and those that would bloom late but will continue in bloom for longer periods (perennials).

Lamiaceae have been used for the provision of ecosystem services such as herbs and spices that provide antioxidants, flavours and food preservatives (Demo et al. 1998; Hossain et al. 2008; Vallverdu-Queralt et al. 2014). Secondary metabolites from the Lamiaceae have activity against human pathogenic organisms (Baydar et al. 2004; Karanika et al. 2001) and have been used for their insecticidal activity against domestic, storage and field crop pests. Extracts of Origanum vulgare L. demonstrated efficacy against $P$. xylostella and Trichoplusia ni Hübner (Lepidoptera: Noctuidae) in a laboratory bioassay (Akhtar and Isman 2004). Ocimum gratissimum L. oil and its constituents have fumigant and repellent activity against a 
number of storage pests (Kim et al. 2003; Ogendo et al. 2008) as well as malaria vectors Aedes aegypti L. (Diptera: Culicidae) and Culex quinquefasciatus Say (Diptera) (Diptera: Culicidae) (Kamaraj et al. 2008).

\section{Outlook and conclusions}

The current review has shown that the most popular habitat manipulation plant families are among the top plant families that have also been exploited for their insecticidal activity. Accordingly, many of the species that have shown benefit in habitat manipulation have unrecognized additional value; they could be exploited for the secondary use of harvesting plant parts to produce botanical insecticides. Habitat manipulation and plant extracts make a potentially effective combination due to the largely benign nature of both tactics on natural enemies. It is widely acknowledged that tactics that can provide multiple ecosystem services may prove effective and most likely to be adopted than are tactics that provide single benefits in isolation (Gentz et al. 2010). The tractability of combining two tactics depends on the effect of each individual tactic on natural enemy populations. For example, an inherent toxicity to natural enemies by a biopesticide would be antagonistic towards conservation biological control. However, a combination in which both botanicals and habitat management do not have negative effects on natural enemies will likely result in additive or synergistic effect. A current study using six non-crop plants including A. conyzoides and Tridax procumbens (Asteraceae) with the same plant for habitat manipulation and plant extracts was successful and cost-effective in managing pests of cabbage (Amoabeng et al. unpublished data).

Much is currently not known about why some plant families have many species useful for both habitat manipulation (pollen and nectar producing) and botanical insecticides. However, there could possibly be link between plants with insecticidal activity being attractive to predators and parasitoids. Secondary metabolites occur in the pollen and nectar and, at optimum concentration, benefit pollinators, parasitoids and predators for example in mediating plant-pollinator interaction, protecting nectar from robbery and other microbial functions such as preserving nutrients in nectar from degradation and reducing diseases in pollen and nectar beneficiaries (Stevenson et al. 2017). It is possible that some plant species in the families producing nectar and with insecticidal activity have common ecological characteristics. According to Campbell (2015), plant defence against herbivores and reproduction do not evolve separately but may have reciprocal and interactive effects on each other. Our hope is that this review will constitute the first step in uniting the separate fields of botanical insecticides and nectar plants for biocontrol enhancement. Future experimental and meta-analysis papers will be required to identify mechanistic patterns that underpin dual utility."

Realising dual or multiple ecosystem services from habitat manipulation is an ambitious call to rapidly restore some lost ecosystem services, but there are other precedents that suggest it is possible, e.g., Davis et al. (2012). Finney et al. (2017) studied the delivery of eight ecosystem services including pests suppression, nitrogen supply, weed suppression among others from ten cover crops. The study showed that where all the plant species supplied biomass, suppressed weeds and retained nitrogen, there were tradeoffs between other ecosystem services among some species. This underscores the need to develop clear understanding of the intended services to be delivered and selection of plant species (Gurr et al. 2017). Ultimately, however, the present review suggests that further research is justified to fully explore scope for using habitat manipulation plants as a source of botanical insecticides. Among the research priorities is field work to assess the phenological and practical issues around duals use. For example, habitat manipulation plants normally need to be established early in the crop calender so they are blooming early in the season, providing resources to natural enemies and thereby prevent pest population build-up. This may be compatible with dual use because harvesting plant parts such as foliage or seed pods for botanical insecticide production could be later in the season on a "needs basis" to deal with uncontrolled pest buildup, should biological control falter. On a wider time scale, habitat manipulation plants could be harvested at the end of the growing season and stored for later processing into botanical insecticides for use in a subsequent crop. In addition, 'non-crop' species may be cultivated on any spare land and harvested for the preparation of botanical insecticides. This would avoid potential disruption of the conservation biological control aspect of the program and might further generate income to individuals who would cultivate plants for the extraction of botanical insecticides. Such low-tech approaches are particularly appropriate for developing country agriculture.

OpenAccess This article is distributed under the terms of the Creative Commons Attribution 4.0 International License (http://creativeco mmons.org/licenses/by/4.0/), which permits unrestricted use, distribution, and reproduction in any medium, provided you give appropriate credit to the original author(s) and the source, provide a link to the Creative Commons license, and indicate if changes were made.

\section{References}

Ahmed S, Zafar MI, Hussain A, Riaz MA, Shahid M (2011) Evaluation of plant extracts on mortality and tunneling activities of 
subterranean termites in Pakistan. In: Stoytcheva M (ed) Pesticides in the modern world: pests control and pesticides exposure and toxicity assessment. InTech, Shanghai, pp 39-54

Ahmed F, Hussein K, Gad M (2015) Biological activity of four plant oils, against the red palm weevil, Rhynchophorus ferrugineus (Oliver), (Coleoptera: Curculionidae). J Biosci Appl Res $1: 213-222$

Aihetasham A, Rasib KZ, Hasan SR, Bodlah I (2017) Effect of Carica papaya, Helianthus annus and Bougainvillea glabra aqueous extracts against termite, Heterotermes indicola (Isoptera: Rhinotermitidae). Punjab Univ J Zool 32:51-56

Akhtar Y, Isman MB (2004) Comparative growth inhibitory and antifeedant effects of plant extracts and pure allelochemicals on four phytophagous insect species. J Appl Entomol 128:32-38. https ://doi.org/10.1046/j.1439-0418.2003.00806.x

Alexenizer M, Dorn A (2007) Screening of medicinal and ornamental plants for insecticidal and growth regulating activity. J Pest Sci 80:205-215. https://doi.org/10.1007/s10340-007-0173-x

Alkan M, Gökçe A, Kara K (2017) Stomach poison activity of some plant extracts on Colorado potato beetle (Coleoptera: Chrysomelidae). Bitki Koruma Bül 57:305-317. https://doi.org/10.16955/ bitkorb.297213

Altieri MA, Ponti L, Nicholls CI (2005) Manipulating vineyard biodiversity for improved insect pest management: case studies from northern California. Int J Biodivers Sci Manage 1:191-203. https ://doi.org/10.1080/17451590509618092

Amoabeng BW, Gurr GM, Gitau CW, Nicol HI, Munyakazi L, Stevenson PC (2013) Tri-trophic insecticidal effects of African plants against cabbage pests. PLoS One 8:e78651. https://doi. org/10.1371/journal.pone.0078651

Amoabeng BW, Gurr GM, Gitau CW, Stevenson PC (2014) Cost:benefit analysis of botanical insecticide use in cabbage: implications for smallholder farmers in developing countries. Crop Prot 57:7176. https://doi.org/10.1016/j.cropro.2013.11.019

Amoabeng B, Asare K, Asare O, Mochiah M, Adama I, Fening K, Gurr G (2017) Pesticides use and misuse in cabbage Brassica oleracea var. capitata L. (Cruciferae) production in Ghana: the influence of farmer education and training. J Agric Ecol Res Int 10:1-9. https://doi.org/10.9734/jaeri/2017/30128

Arnoabeng BW, Stevenson PC, Pandey S, Mochiah MB, Gurr MG (2018) Insecticidal activity of a native Australian tobacco, Nicotiana megalosiphon Van Heurck \& Muell. Arg. (Solanales: Solanaceae) against key insect pests of brassicas. Crop Prot 106:6-12. https://doi.org/10.1016/j.cropro.2017.11.018

Attia S, Grissa KL, Lognay G, Heuskin S, Mailleux AC, Hance T (2011) Chemical composition and acaricidal properties of Deverra scoparia essential oil (Araliales: Apiaceae) and blends of its major constituents against Tetranychus urticae (Acari: Tetranychidae). J Econ Entomol 104:1220-1228. https://doi. org/10.1603/EC10318

Azad AK, Sardar A, Yesmin N, Rahman M, Islam S (2013) Ecofriendly pest control in cucumber (Cucumis sativa L.) field with botanical pesticides. Nat Res 4:404-409. https://doi.org/10.4236/ nr.2013.45050

Babri RA, Khokhar I, Mahmood Z, Mahmud S (2012) Chemical composition and insecticidal activity of the essential oil of Anethum graveolens L. Sci Int (Lahore) 24:453-455

Baggen LR, Gurr GM, Meats A (1999) Flowers in tri-trophic systems: mechanisms allowing selective exploitation by insect natural enemies for conservation biological control. Entomol Exp Appl 91:155-161. https://doi.org/10.1046/j.1570-7458.1999.00478.x

Bale JS, van Lenteren JC, Bigler F (2008) Biological control and sustainable food production. Philos Trans R Soc Lond Ser B Biol Sci 363:761-776. https://doi.org/10.1098/rstb.2007.2182

Balzan MV, Bocci G, Moonen AC (2014) Augmenting flower trait diversity in wildflower strips to optimise the conservation of arthropod functional groups for multiple agroecosystem services. J Insect Conserv 18:713-728. https://doi.org/10.1007/ s10841-014-9680-2

Barzman M, Barberi P, Birch ANE, Boonekamp P, Dachbrodt-Saaydeh S, Graf B, Hommel B, Jensen JE, Kiss J, Kudsk P, Lamichhane JR, Messean A, Moonen AC, Ratnadass A, Ricci P, Sarah JL, Sattin M (2015) Eight principles of integrated pest management. Agron Sustain Dev 35:1199-1215. https://doi.org/10.1007/s1359 3-015-0327-9

Batista MC, Fonseca MCM, Teodoro AV, Martins EF, Pallini A, Venzon M (2017) Basil (Ocimum basilicum L.) attracts and benefits the green lacewing Ceraeochrysa cubana Hagen. Biol Control 110:98-106. https://doi.org/10.1016/j.biocontrol.2017.04.013

Baydar H, Sagdic O, Ozkan G, Karadogan T (2004) Antibacterial activity and composition of essential oils from Origanum, Thymbra and Satureja species with commercial importance in Turkey. Food Control 15:169-172. https://doi.org/10.1016/S0956 -7135(03)00028-8

Begg GS, Cook SM, Dye R, Ferrante M, Franck P, Lavigne C, Lovei GL, Mansion-Vaquie A, Pell JK, Petit S, Quesada N, Ricci B, Wratten SD, Birch ANE (2017) A functional overview of conservation biological control. Crop Prot 97:145-158. https://doi. org/10.1016/j.cropro.2016.11.008

Belmain SR, Amoah BA, Nyirenda SP, Kamanula JF, Stevenson PC (2012) Highly variable insect control efficacy of Tephrosia vogelii chemotypes. J Agric Food Chem 60:10055-10063. https ://doi.org/10.1021/jf3032217

Berenbaum MR, Nitao JK, Zangerl AR (1991) Adaptive significance of furanocoumarin diversity in Pastinaca sativa (Apiaceae). J Chem Ecol 17:207-215. https://doi.org/10.1007/BF00994434

Bhathal S, Singh D, Dhillon R (1994) Insecticidal activity of Ageratum conyzoides Linn. against Lipaphis erysimi (Kaltenbach). J Insect Sci (India) 7:35-36

Blust MH, Hopkins TL (1987) Olfactory responses of a specialist and a generalist grasshopper to volatiles of Artemisia ludoviciana nutt. (Asteraceae). J Chem Ecol 13:1893-1902. https://doi. org/10.1007/BF01013238

Bommarco R, Kleijn D, Potts SG (2013) Ecological intensification: harnessing ecosystem services for food security. Trends Ecol Evol 28:230-238. https://doi.org/10.1016/j.tree.2012.10.012

Borel B (2017) CRISPR, microbes and more are joining the war against crop killers. Nature 543:302-304. https://doi.org/10.1038/54330 $2 \mathrm{a}$

Boulogne I, Petit P, Ozier-Lafontaine H, Desfontaines L, LorangerMerciris G (2012) Insecticidal and antifungal chemicals produced by plants: a review. Environ Chem Lett 10:325-347. https ://doi.org/10.1007/s10311-012-0359-1

Boussaada O, Kamel MBH, Ammar S, Haouas D, Mighri Z, Helal AN (2008) Insecticidal activity of some Asteraceae plant extracts against Tribolium confusum. Bull Insectol 61:283-289

Campbell SA (2015) Ecological mechanisms for the coevolution of mating systems and defence. New Phytol 205:1047-1053

Campbell AJ, Wilby A, Sutton P, Wäckers FL (2017) Do sown flower strips boost wild pollinator abundance and pollination services in a spring-flowering crop? A case study from UK cider apple orchards. Agric Ecosyst Environ 239:20-29. https://doi. org/10.1016/j.agee.2017.01.005

Chagnon M, Kreutzweiser D, Mitchell EA, Morrissey CA, Noome DA, Van der Sluijs JP (2015) Risks of large-scale use of systemic insecticides to ecosystem functioning and services. Environ Sci Pollut Res Int 22:119-134. https://doi.org/10.1007/s1135 6-014-3277-x

Chermenskaya TD, Stepanycheva EA, Shchenikova AV, Chakaeva AS (2010) Insectoacaricidal and deterrent activities of extracts of Kyrgyzstan plants against three agricultural pests. Ind Crops Prod 32:157-163. https://doi.org/10.1016/j.indcrop.2010.04.009 
Christenhusz MJM, Byng JW (2016) The number of known plants species in the world and its annual increase. Phytotaxa 261:201-217. https://doi.org/10.11646/phytotaxa.261.3.1

Chu SS, Liu QR, Liu ZL (2010) Insecticidal activity and chemical composition of the essential oil of Artemisia vestita from China against Sitophilus zeamais. Biochem Syst Ecol 38:489-492. https ://doi.org/10.1016/j.bse.2010.04.011

Cosimi S, Rossi E, Cioni PL, Canale A (2009) Bioactivity and qualitative analysis of some essential oils from Mediterranean plants against stored-product pests: evaluation of repellency against Sitophilus zeamais Motschulsky, Cryptolestes ferrugineus (Stephens) and Tenebrio molitor (L.). J Stored Prod Res 45:125-132. https://doi.org/10.1016/j.jspr.2008.10.002

Dancewicz K, Kordan B, Szumny A, Gabryś B (2012) Aphid behaviour-modifying activity of essential oils from Lamiaceae and Apiaceae. Aphids Other Homopterous Insects 18:93-100

Davis AS, Hill JD, Chase CA, Johanns AM, Liebman M (2012) Increasing cropping system diversity balances productivity, profitability and environmental health. PLoS One 7:e47149. https:// doi.org/10.1371/journal.pone.0047149

de Souza Tavares W, Cruz I, Petacci F, de Assis Júnior SL, de Sousa Freitas S, Zanuncio JC, Serrão JE (2009) Potential use of Asteraceae extracts to control Spodoptera frugiperda (Lepidoptera: Noctuidae) and selectivity to their parasitoids Trichogramma pretiosum (Hymenoptera: Trichogrammatidae) and Telenomus remus (Hymenoptera: Scelionidae). Ind Crops Prod 30:384-388. https://doi.org/10.1016/j.indcrop.2009.07.007

Del-Vechio-Vieira G, Sousa OV, Yamamoto CH, Kaplan MAC (2009) Chemical composition and antimicrobial activity of the essential oils of Ageratum fastigiatum (Asteraceae). Rec Nat Prod 3:52-57

Demo A, Petrakis C, Kefalas P, Boskou D (1998) Nutrient antioxidants in some herbs and Mediterranean plant leaves. Food Res Int 31:351-354. https://doi.org/10.1016/S0963-9969(98)00086-6

Desneux N, Decourtye A, Delpuech JM (2007) The sublethal effects of pesticides on beneficial arthropods. Annu Rev Entomol 52:81106. https://doi.org/10.1146/annurev.ento.52.110405.091440

Deutsch CA, Tewksbury JJ, Tigchelaar M, Battisti DS, Merrill SC, Huey RB, Naylor RL (2018) Increase in crop losses to insect pests in a warming climate. Science 361:916-919. https://doi. org/10.1126/science.aat3466

Diaz MF, Ramirez A, Poveda K (2012) Efficiency of different egg parasitoids and increased floral diversity for the biological control of noctuid pests. Biol Control 60:182-191. https://doi. org/10.1016/j.biocontrol.2011.11.001

Dubey N, Shukla R, Kumar A, Singh P, Prakash B (2011) Global scenario on the application of natural products in integrated pest management programmes. In: Dubey NK (ed) Natural products in plant pest management. CAB International, Wallingford, pp $1-20$

Ebadollahi A (2011) Antifeedant activity of essential oils from Eucalyptus globulus Labill and Lavandula stoechas L. on Tribolium castaneum Herbst (Coleoptera: Tenebrionidae). Biharean Biol 5:8-10

Ebadollahi A (2013) Plant essential oils from apiaceae family as alternatives to conventional insecticides. Ecol Balkan 5:149-172

Eddleston M, Karalliedde L, Buckley N, Fernando R, Hutchinson G, Isbister G, Konradsen F, Murray D, Piola JC, Senanayake N, Sheriff R, Singh S, Siwach SB, Smit L (2002) Pesticide poisoning in the developing world-a minimum pesticides list. Lancet 360:1163-1167. https://doi.org/10.1016/s0140-6736(02)11204-9

Ehler L (1998) Conservation biological control. In: Barbosa P (ed) Past, present, and future: conservation biological control. Academic, San Diego, pp 1-8

El-Ghar GA, Khalil M, Eid T (1996) Some biochemical effects of plant extracts in the black cutworm, Agrotis ipsilon (Hufnagel)
(Lep., Noctuidae). J Appl Entomol 120:477-482. https://doi. org/10.1111/j.1439-0418.1996.tb01638.x

Evergetis E, Haroutounian SA (2014) Exploitation of apiaceae family plants as valuable renewable source of essential oils containing crops for the production of fine chemicals. Ind Crops Prod 54:70-77. https://doi.org/10.1016/j.indcrop.2014.01.009

Fiedler AK, Landis DA, Wratten SD (2008) Maximizing ecosystem services from conservation biological control: the role of habitat management. Biol Control 45:254-271. https://doi.org/10.1016/j. biocontrol.2007.12.009

Finney DM, Murrell EG, White CM, Baraibar B, Barbercheck ME, Bradley BA, Cornelisse S, Hunter MC, Kaye JP, Mortensen DA, Mullen CA, Schipanski ME (2017) Ecosystem services and disservices are bundled in simple and diverse cover cropping systems. Agric Environ Lett 2:170033. https://doi.org/10.2134/ ael2017.09.0033

Frank SD, Shrewsbury PM, Esiekpe O (2008) Spatial and temporal variation in natural enemy assemblages on Maryland native plant species. Environ Entomol 37:478-486. https://doi. org/10.1603/0046-225x(2008)37[478:Satvin]2.0.Co;2

Gbolade AA, Onayade OA, Ayinde BA (2011) Insecticidal activity of Ageratum conyzoides L. volatile oil against Callosobruchus maculatus $\mathrm{F}$. in seed treatment and fumigation laboratory tests. Int J Trop Insect Sci 19:237-240. https://doi.org/10.1017/s1742 758400019536

Gentz MC, Murdoch G, King GF (2010) Tandem use of selective insecticides and natural enemies for effective, reduced-risk pest management. Biol Control 52:208-215. https://doi.org/10.1016/j. biocontrol.2009.07.012

Gill KA, Cox R, O’Neal ME (2014) Quality over quantity: buffer strips can be improved with select native plant species. Environ Entomol 43:298-311. https://doi.org/10.1603/EN13027

Glisic SB, Misic DR, Stamenic MD, Zizovic IT, Asanin RM, Skala DU (2007) Supercritical carbon dioxide extraction of carrot fruit essential oil: chemical composition and antimicrobial activity. Food Chem 105:346-352. https://doi.org/10.1016/j.foodc hem.2006.11.062

Gokce A, Whalon M, Demirtas I, Goren N (2016) Insecticidal compositions and uses thereof. Google Patents

Gontijo LM, Beers EH, Snyder WE (2013) Flowers promote aphid suppression in apple orchards. Biol Control 66:8-15. https://doi. org/10.1016/j.biocontrol.2013.03.007

Griffiths GJK, Holland JM, Bailey A, Thomas MB (2008) Efficacy and economics of shelter habitats for conservation biological control. Biol Control 45:200-209. https://doi.org/10.1016/j.bioco ntrol.2007.09.002

Guarrera PM (1999) Traditional antihelmintic, antiparasitic and repellent uses of plants in Central Italy. J Ethnopharmacol 68:183192. https://doi.org/10.1016/S0378-8741(99)00089-6

Guillet G, Philogene BJR, Omeara J, Durst T, Arnason JT (1997) Multiple modes of insecticidal action of three classes of polyacetylene derivatives from Rudbeckia hirta. Phytochemistry 46:495498. https://doi.org/10.1016/s0031-9422(97)00320-8

Gurr GM, Barlow ND, Memmott J, Wratten SD, Greathead DJ (2000a) A history of methodological, theoretical and empirical approaches to biological control. In: Gurr G, Wratten S (eds) Biological control: measures of success. Springer, Dordrecht, pp 3-37

Gurr GM, Wratten SD, Barbosa P (2000b) Success in conservation biological control of arthropods. In: Gurr G, Wratten S (eds) Biological control: measures of success. Springer, Dordrecht, pp 105-132

Gurr GM, Lu Z, Zheng X, Xu H, Zhu P, Chen G, Yao X, Cheng J, Zhu Z, Catindig JL, Villareal S, Van Chien H, le Cuong Q, Channoo C, Chengwattana N, Lan LP, le Hai H, Chaiwong J, Nicol HI, Perovic DJ, Wratten SD, Heong KL (2016) Multi-country 
evidence that crop diversification promotes ecological intensification of agriculture. Nat Plants 2:16014. https://doi.org/10.1038/ nplants.2016.14

Gurr GM, Wratten SD, Landis DA, You M (2017) Habitat management to suppress pest populations: progress and prospects. Annu Rev Entomol 62:91-109. https://doi.org/10.1146/annurev-ento-03161 6-035050

Gurr GM, Reynolds OL, Johnson AC, Desneux N, Zalucki MP, Furlong MJ, Li ZY, Akutse KS, Chen JH, Gao XW, You MS (2018) Landscape ecology and expanding range of biocontrol agent taxa enhance prospects for diamondback moth management. A review. Agron Sustain Dev 38:23. https://doi.org/10.1007/s1359 3-018-0500-Z

Harley RM, Atkins S, Budantsev AL, Cantino PD, Conn BJ, Grayer R, Harley MM, De Kok RD, Krestovskaja TD, Morales R (2004) Labiatae. In: Kadereit JW (ed) Flowering plants dicotyledons. Springer, Berlin, pp 167-275

Haro MM, Silveira LCP, Wilby A (2018) Stability lies in flowers: plant diversification mediating shifts in arthropod food webs. PLoS One 13:e0193045. https://doi.org/10.1371/journal.pone.0193045

Hasheminia SM, Sendi JJ, Jahromi KT, Moharramipour S (2011) The effects of Artemisia annиa L. and Achillea millefolium L. crude leaf extracts on the toxicity, development, feeding efficiency and chemical activities of small cabbage Pieris rapae L. (Lepidoptera: Pieridae). Pestic Biochem Physiol 99:244-249. https://doi. org/10.1016/j.pestbp.2010.12.009

Hatt S, Lopes T, Boeraeve F, Chen JL, Francis F (2017) Pest regulation and support of natural enemies in agriculture: experimental evidence of within field wildflower strips. Ecol Eng 98:240-245. https://doi.org/10.1016/j.ecoleng.2016.10.080

Heywood VH, Brummitt RK, Culham A, Seberg O (2007) Flowering plant families of the world. Kew, London

Hossain M, Haque M (2010) Efficacy of some indigenous plant extracts as grain protectant against pulse beetle, Callosobruchus chinensis L. J Agro For Environ 4:197-202

Hossain M, Brunton N, Barry-Ryan C, Martin-Diana AB, Wilkinson M (2008) Antioxidant activity of spice extracts and phenolics in comparison to synthetic antioxidants. Rasayan J Chem 1:751756. https://doi.org/10.21427/D7105D

Isman MB (1997) Neem and other botanical insecticides: barriers to commercialization. Phytoparasitica 25:339-344. https://doi. org/10.1007/Bf02981099

Isman MB (2000) Plant essential oils for pest and disease management. Crop Prot 19:603-608. https://doi.org/10.1016/S0261 -2194(00)00079-X

Isman MB (2006) Botanical insecticides, deterrents, and repellents in modern agriculture and an increasingly regulated world. Annu Rev Entomol 51:45-66. https://doi.org/10.1146/annur ev.ento.51.110104.151146

Isman MB (2008) Botanical insecticides: for richer, for poorer. Pest Manag Sci 64:8-11. https://doi.org/10.1002/ps.1470

Isman MB (2017) Bridging the gap: moving botanical insecticides from the laboratory to the farm. Ind Crops Prod 110:10-14. https://doi. org/10.1016/j.indcrop.2017.07.012

Isman MB, Grieneisen ML (2014) Botanical insecticide research: many publications, limited useful data. Trends Plant Sci 19:140-145. https://doi.org/10.1016/j.tplants.2013.11.005

Jbilou R, Amri H, Bouayad N, Ghailani N, Ennabili A, Sayah F (2008) Insecticidal effects of extracts of seven plant species on larval development, alpha-amylase activity and offspring production of Tribolium castaneum (Herbst) (Insecta: Coleoptera: Tenebrionidae). Bioresour Technol 99:959-964. https://doi.org/10.1016/j. biortech.2007.03.017

Kamaraj C, Rahuman AA, Bagavan A (2008) Antifeedant and larvicidal effects of plant extracts against Spodoptera litura (F.),
Aedes aegypti L. and Culex quinquefasciatus Say. Parasitol Res 103:325-331. https://doi.org/10.1007/s00436-008-0974-8

Karanika MS, Komaitis M, Aggelis G (2001) Effect of aqueous extracts of some plants of Lamiaceae family on the growth of Yarrowia lipolytica. Int J Food Microbiol 64:175-181. https:// doi.org/10.1016/S0168-1605(00)00468-2

Karp DS, Chaplin-Kramer R, Meehan TD, Martin EA, DeClerck F, Grab H, Gratton C, Hunt L, Larsen AE, Martinez-Salinas A, O’Rourke ME, Rusch A, Poveda K, Jonsson M, Rosenheim JA, Schellhorn NA, Tscharntke T, Wratten SD, Zhang W, Iverson AL, Adler LS, Albrecht M, Alignier A, Angelella GM, Zubair Anjum M, Avelino J, Batary P, Baveco JM, Bianchi F, Birkhofer K, Bohnenblust EW, Bommarco R, Brewer MJ, Caballero-Lopez B, Carriere Y, Carvalheiro LG, Cayuela L, Centrella M, Cetkovic A, Henri DC, Chabert A, Costamagna AC, De la Mora A, de Kraker J, Desneux N, Diehl E, Diekotter T, Dormann CF, Eckberg JO, Entling MH, Fiedler D, Franck P, Frank van Veen FJ, Frank T, Gagic V, Garratt MPD, Getachew A, Gonthier DJ, Goodell PB, Graziosi I, Groves RL, Gurr GM, HajianForooshani Z, Heimpel GE, Herrmann JD, Huseth AS, Inclan DJ, Ingrao AJ, Iv P, Jacot K, Johnson GA, Jones L, Kaiser M, Kaser JM, Keasar T, Kim TN, Kishinevsky M, Landis DA, Lavandero B, Lavigne C, Le Ralec A, Lemessa D, Letourneau DK, Liere H, Lu Y, Lubin Y, Luttermoser T, Maas B, Mace K, Madeira F, Mader V, Cortesero AM, Marini L, Martinez E, Martinson HM, Menozzi P, Mitchell MGE, Miyashita T, Molina GAR, MolinaMontenegro MA, O'Neal ME, Opatovsky I, Ortiz-Martinez S, Nash M, Ostman O, Ouin A, Pak D, Paredes D, Parsa S, Parry H, Perez-Alvarez R, Perovic DJ, Peterson JA, Petit S, Philpott SM, Plantegenest M, Plecas M, Pluess T, Pons X, Potts SG, Pywell RF, Ragsdale DW, Rand TA, Raymond L, Ricci B, Sargent C, Sarthou JP, Saulais J, Schackermann J, Schmidt NP, Schneider G, Schuepp C, Sivakoff FS, Smith HG, Stack Whitney K, Stutz S, Szendrei Z, Takada MB, Taki H, Tamburini G, Thomson LJ, Tricault Y, Tsafack N, Tschumi M, Valantin-Morison M, Van Trinh M, van der Werf W, Vierling KT, Werling BP, Wickens JB, Wickens VJ, Woodcock BA, Wyckhuys K, Xiao H, Yasuda M, Yoshioka A, Zou Y (2018) Crop pests and predators exhibit inconsistent responses to surrounding landscape composition. Proc Natl Acad Sci USA 115:E7863-E7870. https://doi. org/10.1073/pnas.1800042115

Khan ZR, Midega CAO, Hassanali A, Pickett JA, Wadhams LJ, Wanjoya A (2006) Management of witchweed, Striga hermonthica, and stemborers in sorghum, Sorghum bicolor, through intercropping with greenleaf desmodium, Desmodium intortum. Int J Pest Manag 52:297-302. https://doi.org/10.1080/096708706008169 91

Khan ZR, Midega CA, Pittchar J, Bruce TJ, Pickett JA (2012) 'PushPull' revisited: The process of successful deployment of a chemical ecology based pest management tool. In: Gurr GM, Wratten SD, Synder WE, Read DMY (eds) Biodiversity and insect pests: key issues for sustainable management. Wiley, Chicheste, pp 259-275

Khater HF, Khater DF (2009) The insecticidal activity of four medicinal plants against the blowfly Lucilia sericata (Diptera: Calliphoridae). Int J Dermatol 48:492-497. https://doi.org/10.111 1/j.1365-4632.2009.03937.x

Kim SI, Roh JY, Kim DH, Lee HS, Ahn YJ (2003) Insecticidal activities of aromatic plant extracts and essential oils against Sitophilus oryzae and Callosobruchus chinensis. J Stored Prod Res 39:293303. https://doi.org/10.1016/S0022-474x(02)00017-6

Koul O (2004) Insect antifeedants. CRC, Boca Raton

Koul O, Walia S, Dhaliwal G (2008) Essential oils as green pesticides: potential and constraints. Biopestic Int 4:63-84

Kozawa M, Baba K, Matsuyama Y, Kido T, Sakai M, Takemoto T (1982) Components of the root of Anthriscus sylvestris HOFFM. 
II. Insecticidal activity. Chem Pharm Bull 30:2885-2888. https ://doi.org/10.1248/cpb.30.2885

Kumar P, Mishra S, Malik A, Satya S (2011) Insecticidal properties of Mentha species: a review. Ind Crops Prod 34:802-817. https:// doi.org/10.1016/j.indcrop.2011.02.019

Kumar R, Ahad I, Sheikh AA, Showkat A, Arifie U, Dorjey S (2017) Bioactive plant extracts an alternate to chemicals for management of armyworm infesting oats. Int J Curr Microbiol Appl Sci 6:129-134. https://doi.org/10.20546/ijcmas.2017.612.017

Larocque N, Vincent C, Belanger A, Bourassa JP (1999) Effects of tansy essential oil from Tanacetum vulgare on biology of oblique-banded leafroller, Choristoneura rosaceana. J Chem Ecol 25:1319-1330. https://doi.org/10.1023/A:1020974725437

Lavandero B, Wratten SD, Didham RK, Gurr G (2006) Increasing floral diversity for selective enhancement of biological control agents: a double-edged sward? Basic Appl Ecol. 7:236-243. https://doi. org/10.1016/j.baae.2005.09.004

Lee SB, Rasmussen SK (2000) Molecular markers in some medicinal plants of the Apiaceae family. Euphytica 114:87-91. https://doi. org/10.1023/A:1003919416122

Lee H-S, Choi G-J, Cho K-Y, Lee S-G, Ahn Y-J (2000) Fungicidal and insecticidal activities of various grain extracts against five insect pests and six phytopathogenic fungi. Korean J Pesti Sci 4:7-14

Lee BH, Annis PC, Tumaalii F, Choi WS (2004) Fumigant toxicity of essential oils from the Myrtaceae family and 1,8-cineole against 3 major stored-grain insects. J Stored Prod Res 40:553-564. https ://doi.org/10.1016/j.jspr.2003.09.001

Li H, Cheng F, Wei Y, Lydy MJ, You J (2017) Global occurrence of pyrethroid insecticides in sediment and the associated toxicological effects on benthic invertebrates: an overview. J Hazard Mater 324:258-271. https://doi.org/10.1016/j.jhazmat.2016.10.056

Liang Y, Li X, Gu Z, Qin P, Ji M (2015) Toxicity of amorphigenin from the seeds of Amorpha fruticosa against the larvae of Culex pipiens pallens (Diptera: Culicidae). Molecules 20:3238-3254. https://doi.org/10.3390/molecules20023238

Lopez MD, Jordan MJ, Pascual-Villalobos MJ (2008) Toxic compounds in essential oils of coriander, caraway and basil active against stored rice pests. J Stored Prod Res 44:273-278. https:// doi.org/10.1016/j.jspr.2008.02.005

Macedo ME, Consoli RAGB, Grandi TSM, dosAnjos AMG, deOliveira AB, Mendes NM, Queiroz RO, Zani CL (1997) Screening of Asteraceae (Compositae) plant extracts for larvicidal activity against Aedes fluviatilis (Diptera: Culicidae). Mem Inst Oswaldo Cruz 92:565-570. https://doi.org/10.1590/S0074-0276199700 0400024

Mansour SA, Ibrahim RM, El-Gengaihi SE (2014) Insecticidal activity of chicory (Cichorium intybus L.) extracts against two dipterous insect-disease vectors: mosquito and housefly. Ind Crops Prod 54:192-202. https://doi.org/10.1016/j.indcrop.2014.01.011

Martinez-Una A, Martin JM, Fernandez-Quintanilla C, Dorado J (2013) Provisioning floral resources to attract aphidophagous hoverflies (Diptera: Syrphidae) useful for pest management in central Spain. J Econ Entomol 106:2327-2335. https://doi. org/10.1603/ec13180

Matasyoh JC, Maiyo ZC, Ngure RM, Chepkorir R (2009) Chemical composition and antimicrobial activity of the essential oil of Coriandrum sativum. Food Chem 113:526-529. https://doi. org/10.1016/j.foodchem.2008.07.097

Maulidiani Abas F, Khatib A, Shaari K, Lajis NH (2014) Chemical characterization and antioxidant activity of three medicinal Apiaceae species. Ind Crops Prod 55:238-247. https://doi. org/10.1016/j.indcrop.2014.02.013

Medhini N, Divakar Y, Manjulakumari D (2012) Effect of Calendula officinalis extracts on the nutrient components of different tissues of tobacco cutworm, Spodoptera litura Fabricius. J Biopestic $5: 139$
Meradsi F, Laamari M (2016) Aphicidal and behavioral effects of Vicia faba L. (Fabales: Fabaceae) leaf extracts against Aphis fabae Scopoli (Hom., Aphididae). Jordan J Agric Sci 405:1-10

Merah O, Djazouli Z-E (2016) Toxicity evaluation of Dittrichia iviscosa L's aqueous extracts in combination with bio-adjuvant Silene fuscata on Chaitophorus leucomelas Koch. (Hom., Aphididae) and on biocenotic resumption of functional groups. Jordan J Agric Sci 12:797-814

Mohankumar TK, Shivanna KS, Achuttan VV (2016) Screening of methanolic plant extracts against larvae of Aedes aegypti and Anopheles stephensi in Mysore. J Arthrop Borne Dis 10:303-314

Momen F, Abdel Rahman H, Samour E, Aly S, Fahim S (2014) Acaricidal activity of Melissa officinalis oil and its formulation on Tetranychus urticae and the predatory mite Neoseiulus californicus (Acari: Tetranychidae and Phytoseiidae). Acta Phytol Entomol Hung 49:95-115

Momin RA, Nair MG (2002) Pest-managing efficacy of trans-asarone isolated from Daucus carota L. seeds. J Agric Food Chem 50:4475-4478. https://doi.org/10.1021/jf020209r

Moreira MD, Picanco MC, Barbosa LCD, Guedes RNC, de Campos MR, Silva GA, Martins JC (2007) Plant compounds insecticide activity against Coleoptera pests of stored products. Pesqui Agropecu Bras 42:909-915. https://doi.org/10.1590/S0100-204x2 007000700001

Morimoto M, Tanimoto K, Sakatani A, Komai K (2002) Antifeedant activity of an anthraquinone aldehyde in Galium aparine L. against Spodoptera litura F. Phytochemistry 60:163-166. https ://doi.org/10.1016/S0031-9422(02)00095-X

Mullin CA, Alfatafta AA, Harman JL, Everett SL, Serino AA (1991) Feeding and toxic effects of floral sesquiterpene lactones, diterpenes, and phenolics from sunflower (Helianthusannuus L.) on Western Corn-Rootworm. J Agric Food Chem 39:2293-2299. https://doi.org/10.1021/jf00012a041

Nave A, Goncalves F, Crespi AL, Campos M, Torres L (2016) Evaluation of native plant flower characteristics for conservation biological control of Prays oleae. Bull Entomol Res 106:249-257. https://doi.org/10.1017/S0007485315001091

Nenaah GE, Ibrahim SIA, Al-Assiuty BA (2015) Chemical composition, insecticidal activity and persistence of three Asteraceae essential oils and their nanoemulsions against Callosobruchus maculatus (F.). J Stored Prod Res 61:9-16. https://doi. org/10.1016/j.jspr.2014.12.007

Ogendo JO, Kostyukovsky M, Ravid U, Matasyoh JC, Deng AL, Omolo EO, Kariuki ST, Shaaya E (2008) Bioactivity of Ocimum gratissimum $\mathrm{L}$. oil and two of its constituents against five insect pests attacking stored food products. J Stored Prod Res 44:328-334. https://doi.org/10.1016/j.jspr.2008.02.009

Okunade AL (2002) Ageratum conyzoides L. (Asteraceae). Fitoterapia 73:1-16. https://doi.org/10.1016/S0367-326X(01)00364-1

Padin SB, Fuse C, Urrutia MI, Dal Bello GM (2013) Toxicity and repellency of nine medicinal plants against Tribolium castaneum in stored wheat. BulInsectol 66:45-49

Pandey S, Rahman A, Gurr GM (2018) Australian native flowering plants enhance the longevity of three parasitoids of brassica pests. Entomol Exp Appl 166:265-276. https://doi.org/10.1111/ eea. 12668

Park IK, Choi KS, Kim DH, Choi IH, Kim LS, Bak WC, Choi JW, Shin SC (2006) Fumigant activity of plant essential oils and components from horseradish (Armoracia rusticana), anise (Pimpinella anisum) and garlic (Allium sativum) oils against Lycoriella ingenиа (Diptera: Sciaridae). Pest Manag Sci 62:723-728. https ://doi.org/10.1002/ps.1228

Pascual-Villalobos MJ, Robledo A (1999) Anti-insect activity of plant extracts from the wild flora in southeastern Spain. Biochem Syst Ecol 27:1-10. https://doi.org/10.1016/S0305-1978(98)00051-9 
Pavela R (2004) Insecticidal activity of certain medicinal plants. Fitoterapia 75:745-749. https://doi.org/10.1016/j.fitote.2004.08.005

Pavela R (2009) Larvicidal effects of some Euro-Asiatic plants against Culex quinquefasciatus Say larvae (Diptera: Culicidae). Parasitol Res 105:887-892. https://doi.org/10.1007/s00436-009-1511-0

Pavela R (2010) Antifeedant activity of plant extracts on Leptinotarsa decemlineata Say. and Spodoptera littoralis Bois. larvae. Ind Crops Prod 32:213-219. https://doi.org/10.1016/j.inder op.2010.04.010

Pavela R (2011) Screening of Eurasian plants for insecticidal and growth inhibition activity against Spodoptera littoralis larvae. Afr J Agric Res 6:2895-2907

Pavela R, Benelli G (2016) Essential oils as ecofriendly biopesticides? Challenges and constraints. Trends Plant Sci 21:1000-1007. https://doi.org/10.1016/j.tplants.2016.10.005

Pavela R, Vrchotova N, Triska J (2016) Larvicidal activity of extracts from Ammi visnaga Linn. (Apiaceae) seeds against Culex quinquefasciatus Say. (Diptera: Culicidae). Exp Parasitol 165:51-57. https://doi.org/10.1016/j.exppara.2016.03.016

Pelah D, Abramovich Z, Markus A, Wiesman Z (2002) The use of commercial saponin from Quillaja saponaria bark as a natural larvicidal agent against Aedes aegypti and Culex pipiens. $\mathrm{J}$ Ethnopharmacol 81:407-409. https://doi.org/10.1016/S0378 -8741(02)00138-1

Pellissier ME, Jabbour R (2018) Herbivore and parasitoid insects respond differently to annual and perennial floral strips in an alfalfa ecosystem. Biol Control 123:28-35. https://doi. org/10.1016/j.biocontro1.2018.04.014

Pretty J, Benton TG, Bharucha ZP, Dicks LV, Flora CB, Godfray HCJ, Goulson D, Hartley S, Lampkin N, Morris C, Pierzynski G, Prasad PVV, Reganold J, Rockstrom J, Smith P, Thorne P, Wratten $S$ (2018) Global assessment of agricultural system redesign for sustainable intensification. Nature Sustain 1:441-446. https ://doi.org/10.1038/s41893-018-0114-0

Raghavendra BS, Prathibha KP, Vijayan VA (2013) Synergistic effect of Eugenia jambolana Linn. and Solidago canadensis Linn. leaf extracts with deltamethrin against the dengue vector Aedes aegypti Linn. at Mysore. Environ Sci Pollut Res 20:3830-3835. https://doi.org/10.1007/s11356-012-1308-z

Rajeswary M, Govindarajan M (2013) Mosquito larvicidal and phytochemical properties of Ageratina adenophora (Asteraceae) against three important mosquitoes. J Vector Borne Dis 50:141-143

Rani PU (2012) Fumigant and contact toxic potential of essential oils from plant extracts against stored product pests. J Biopestic 5:120

Regnault-Roger C, Hamraoui A, Holeman M, Theron E, Pinel R (1993) Insecticidal effect of essential oils from mediterranean plants upon Acanthoscelides obtectus Say (Coleoptera, Bruchidae), a pest of kidney bean (Phaseolus vulgaris L.). J Chem Ecol 19:1233-1244. https://doi.org/10.1007/BF00987383

Regnault-Roger C, Ribodeau M, Hamraoui A, Bareaua I, Blanchard P, Gil-Munoz MI, Barberan FT (2004) Polyphenolic compounds of Mediterranean Lamiaceae and investigation of orientational effects on Acanthoscelides obtectus (Say). J Stored Prod Res 40:395-408. https://doi.org/10.1016/S0022-474x(03)00031-6

Rizvi SAH, Ling SQ, Tian FJ, Xie F, Zeng XNA (2018) Toxicity and enzyme inhibition activities of the essential oil and dominant constituents derived from Artemisia absinthium L. against adult Asian citrus psyllid Diaphorina citri Kuwayama (Hemiptera: Psyllidae). Ind Crops Prod 121:468-475. https://doi. org/10.1016/j.indcrop.2018.05.031

Rockstrom J, Steffen W, Noone K, Persson A, Chapin FS, Lambin E, Lenton TM, Scheffer M, Folke C, Schellnhuber HJ, Nykvist B, de Wit CA, Hughes T, van der Leeuw S, Rodhe H, Sorlin S, Snyder PK, Costanza R, Svedin U, Falkenmark M, Karlberg L, Corell RW, Fabry VJ, Hansen J, Walker B, Liverman D, Richardson K,
Crutzen P, Foley J (2009) Planetary boundaries: exploring the safe operating space for humanity. Ecol Soc 14:32

Sampson BJ, Tabanca N, Kirimer N, Demirci B, Baser KH, Khan IA, Spiers JM, Wedge DE (2005) Insecticidal activity of 23 essential oils and their major compounds against adult Lipaphis pseudobrassicae (Davis) (Aphididae: Homoptera). Pest Manag Sci 61:1122-1128. https://doi.org/10.1002/ps.1100

Santos PM, Figueiredo AC, Oliveira MM, Barroso JG, Pedro LG, Deans SG, Younus AKM, Scheffer JC (1998) Essential oils from hairy root cultures and from fruits and roots of Pimpinella anisum. Phytochem 48:455-460. https://doi.org/10.1016/S0031 -9422(98)00022-3

Schmid R (2004) Reviews and notices of publications. Taxon 53:1117-1126

Senatore F, Napolitano F, Mohamed MAH, Harris PJC, Minkeni PNS, Henderson J (2004) Antibacterial activity of Tagetes minuta L. (Asteraceae) essential oil with different chemical composition. Flav Frag J 19:574-578. https://doi.org/10.1002/ffj.1358

Sibul I, Luik A, Voolma K (2001) Possibilities to influence maturation feeding of the large pine weevil, Hylobius abietis L., with plant extracts and neem preparations. In: Proceedings of the international workshop. Estonian Agricultural University, Inst. of Plant Protection, Tartu, Estonia

Sivaraman G, Paulraj MG, Ignacimuthu S, Al-Dhabi N (2014) Bioefficacy of Cleome viscosa L. and Sinapis alba L. seed extracts against Helicoverpa armigera (Hubner) (Lepidoptera: Noctuidae). Int J Pure Appl Zool 2:211-217

Sivinski J (2014) The attraction of lepidoptera to flowering plants also attractive to parasitoids (Diptera, Hymenoptera). Florida Entomol 97:1317-1327. https://doi.org/10.1653/024.097.0406

Sousa RM, Rosa JS, Oliveira L, Cunha A, Fernandes-Ferreira M (2013) Activities of Apiaceae essential oils against armyworm, Pseudaletia unipuncta (Lepidoptera: Noctuidae). J Agric Food Chem 61:7661-7672. https://doi.org/10.1021/jf403096d

Sousa RMOF, Rosa JS, Oliveira L, Cunha A, Fernandes-Ferreira M (2015) Activities of Apiaceae essential oils and volatile compounds on hatchability, development, reproduction and nutrition of Pseudaletia unipuncta (Lepidoptera: Noctuidae). Ind Crops Prod 63:226-237. https://doi.org/10.1016/j.indcrop.2014.09.052

Stevens PF, Davis H (2001) Angiosperm phylogeny. University of Missouri, St Louis

Stevenson PC, Nicolson SW, Wright GA (2017) Plant secondary metabolites in nectar: impacts on pollinators and ecological functions. Funct Ecol 31:65-75

Struik PC, Kuyper T (2017) Sustainable intensification in agriculture: the richer shade of green. A review. Agron Sustain Dev. https:// doi.org/10.1007/s13593-017-0445-7

Sumon KA, Ritika AK, Peeters E, Rashid H, Bosma RH, Rahman MS, Fatema MK, Van den Brink PJ (2018) Effects of imidacloprid on the ecology of sub-tropical freshwater microcosms. Environ Pollut 236:432-441. https://doi.org/10.1016/j.envpol.2018.01.102

Tabanca N, Bernier UR, Tsikolia M, Becnel JJ, Sampson B, Werle C, Demirci B, Baser KH, Blythe EK, Pounders C, Wedge DE (2010) Eupatorium capillifolium essential oil: chemical composition, antifungal activity, and insecticidal activity. Nat Prod Comm 5:1409-1415

Tembo Y, Mkindi AG, Mkenda PA, Mpumi N, Mwanauta R, Stevenson PC, Ndakidemi PA, Belmain SR (2018) Pesticidal plant extracts improve yield and reduce insect pests on legume crops without harming beneficial arthropods. Front Plant Sci 9:1425. https:// doi.org/10.3389/fpls.2018.01425

Tilman D, Balzer C, Hill J, Befort BL (2011) Global food demand and the sustainable intensification of agriculture. Proc Natl Acad Sci USA 108:20260-20264. https://doi.org/10.1073/pnas.11164 37108 
Trisnawati I, Azis A (2017) The effectiveness of habitat modification schemes for enhancing beneficial insects: assessing the importance of trap cropping management approach, vol. 1854. In: Murkovic M, Risuleo C, Prasetyo EN, Shovitri M, Nyanhongo GS (eds) Proceeding of international biology conference 2016: biodiversity and biotechnology for human welfare

Tscharntke T, Bommarco R, Clough Y, Crist TO, Kleijn D, Rand TA, Tylianakis JM, van Nouhuys S, Vidal S (2007) Conservation biological control and enemy diversity on a landscape scale. Biol Control 43:294-309. https://doi.org/10.1016/j.biocontrol .2007 .08 .006

Tschumi M, Albrecht M, Collatz J, Dubsky V, Entling MH, Najar-Rodriguez AJ, Jacot K (2016) Tailored flower strips promote natural enemy biodiversity and pest control in potato crops. J Appl Ecol 53:1169-1176. https://doi.org/10.1111/1365-2664.12653

Tuell JK, Fiedler AK, Landis D, Isaacs R (2008) Visitation by wild and managed bees (Hymenoptera: Apoidea) to Eastern U.S. native plants for use in conservation programs. Environ Entomol 37:707-718. https://doi.org/10.1603/0046225x(2008)37[707:Vbwamb]2.0.Co;2

Vallverdu-Queralt A, Regueiro J, Martinez-Huelamo M, Rinaldi Alvarenga JF, Leal LN, Lamuela-Raventos RM (2014) A comprehensive study on the phenolic profile of widely used culinary herbs and spices: rosemary, thyme, oregano, cinnamon, cumin and bay. Food Chem 154:299-307. https://doi.org/10.1016/j.foodc hem.2013.12.106

van Rijn PCJ, Wäckers FL, Cadotte M (2016) Nectar accessibility determines fitness, flower choice and abundance of hoverflies that provide natural pest control. J Appl Ecol 53:925-933. https ://doi.org/10.1111/1365-2664.12605

Vattala HD, Wratten SD, Phillips CB, Wackers FL (2006) The influence of flower morphology and nectar quality on the longevity of a parasitoid biological control agent. Biol Cont 39:179-185. https://doi.org/10.1016/j.biocontrol.2006.06.003

Villa M, Marrão R, Mexia A, Bento A, Pereira JA (2016) Are wild flowers and insect honeydews potential food resources for adults of the olive moth, Prays oleae? J Pest Sci 90:185-194. https:// doi.org/10.1007/s10340-016-0745-8

Vukajlović FN, Pešić SB, Tanasković ST, Predojević DZ, Gvozdenac SM, Prvulović DM, Bursić VP (2018) Efficacy of Echium spp. water extracts as post-harvest grain protectants against Plodia interpunctella. Roman Biotechnol Lett. https://doi.org/10.26327 /rbl2017.102 (in press)

Walton NJ, Isaacs R (2011) Influence of native flowering plant strips on natural enemies and herbivores in adjacent blueberry fields. Environ Entomol 40:697-705. https://doi.org/10.1603/EN10288

Williamson S, Ball A, Pretty J (2008) Trends in pesticide use and drivers for safer pest management in four African countries. Crop Prot 27:1327-1334. https://doi.org/10.1016/j.cropro.2008.04.006

Wyckhuys KAG, Lu YH, Morales H, Vazquez LL, Legaspi JC, Eliopoulos PA, Hernandez LM (2013) Current status and potential of conservation biological control for agriculture in the developing world. Biol Control 65:152-167. https://doi.org/10.1016/j. biocontrol.2012.11.010

Xu HX, Yang YJ, Lu YH, Zheng XS, Tian JC, Lai FX, Fu Q, Lu ZX (2017) Sustainable management of rice insect pests by nonchemical-insecticide technologies in China. Rice Sci 24:61-72. https://doi.org/10.1016/j.rsci.2017.01.001

Yang RZ, Tang CS (1988) Plants used for pest-control in china-a literature-review. Econ Bot 42:376-406. https://doi.org/10.1007/ Bf02860162

Yeom HJ, Jung CS, Kang J, Kim J, Lee JH, Kim DS, Kim HS, Park PS, Kang KS, Park IK (2015) Insecticidal and acetylcholine esterase inhibition activity of Asteraceae plant essential oils and their constituents against adults of the German cockroach (Blattella germanica). J Agric Food Chem 63:2241-2248. https://doi. org/10.1021/jf505927n 